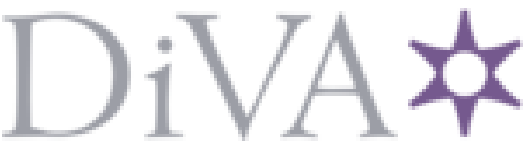

http://www.diva-portal.org

\title{
Postprint
}

This is the accepted version of a paper published in IEEE Transactions on Cybernetics. This paper has been peer-reviewed but does not include the final publisher proof-corrections or journal pagination.

Citation for the original published paper (version of record):

Sun, D., Naghdy, F., Du, H. (2017)

Neural Network-Based Passivity Control of Teleoperation System Under Time-Varying

Delays.

IEEE Transactions on Cybernetics, 47(7): 1666-1680

https://doi.org/10.1109/TCYB.2016.2554630

Access to the published version may require subscription.

N.B. When citing this work, cite the original published paper.

Permanent link to this version:

http://urn.kb.se/resolve?urn=urn:nbn:se:oru:diva-64476 


\section{Neural Network based Passivity Control of Teleoperation System under Time-Varying Delays}

Da Sun

Faculty of Engineering and Information sciences,

University of Wollongong

\author{
Fazel Naghdy \\ Faculty of Engineering and Information sciences, \\ University of Wollongong
}

\author{
Haiping Du \\ Faculty of Engineering and Information sciences, \\ University of Wollongong
}

\begin{abstract}
In this paper, a novel neural network-based fourchannel wave-based Time Domain Passivity approach (TDPA) is proposed for a teleoperation system with time-varying delays. The designed wave-based TDPA aims to robustly guarantee the channels passivity and provide higher transparency than the previous power-based TDPA. The applied neural network is used to estimate and eliminate the system's dynamic uncertainties. The system stability with linearity assumption on human and environment has been analyzed using Lyapunov method. The proposed algorithm is validated through experimental work based on a 3-DOF bilateral teleoperation platform in the presence of different time delays.
\end{abstract}

Index Terms-Bilateral teleoperation, Neural network, Wave variable, TDPA, Passivity, Time-varying delays.

\section{Introduction}

In the last five decades, teleoperation technologies have been widely applied and developed all around the world. Teleoperation system is defined as electromechanical mechanism that extends human's sensing, decision making and manipulation capability to the remote environment. A conventional teleoperation system consists of the human operator, the master robot, the communication networks, the slave robot and the environmental tasks. Teleoperation systems have numerous applications ranging from space exploration [1], underwater operation [2], mining [3], nuclear reactor [4] where human operators are protected from dangerous situations, to medical training [5], rehabilitation [6] and minimally invasive surgery [7] where a patient suffers less trauma through key-hole surgery. A teleoperation system is called unilateral if only the master's control signals are transmitted to the slave side. If there exists the motion or force feedback from the slave side to the master, this system can be called bilateral. Bilateral teleoperation is assessed through the two critical indices of stability and transparency. Stability requires the closed loop system to be stable under different environmental conditions. Ideal transparency means that the medium between the operator and the environment is not felt and the dynamics of the master and the slave are canceled out.

With the network technologies advancing at a staggering rate, teleoperation can be conducted by using commercially available communication networks. When the local and remote platforms are connected via commercial networks, the forward and feedback control signals between the master and the slave will be inevitably associated with time delays. In remote control and manipulation, without proper control algorithms, even a small time delay may destabilize and degrade the tracking performance of a teleoperation system. Numerous methods have been proposed to balance the trade-off between the system stability and transparency in the presence of time delays. A system designed by Lee and Spong uses direct position feedback to eliminate position drift [8]. Nunõ et al. deploy P-like, PD-like and scattering controllers to analyze the stability of the nonlinear teleoperation systems with the classic assumptions of passivity [9]. Later, they introduce a general Lyapunov-like function to unify stability analysis on the passivity-based control for the nonlinear teleoperation systems [10]. An adaptive coordination control law based on the scattering approach is introduced by Chopra et al. to ensure position synchronization in the nonlinear teleoperation systems [11]. Yang et al. design a new fuzzy PDlike controller to deal with uncertainties of system dynamics [12]. However, all of the P-like and PD-like systems require pre-set dampers with constant gains associated with the value of time delays to guarantee the system stability by reducing transparency. Due to different types of time delays, these methods may be overconservative in some situations.

In recent times, the neural networks $(\mathrm{NN})$ have attracted much attention due to their prominent properties such as learning capability mapping and parallel processing. NNs have been deployed in the control of the robotic systems and have significantly improved their performance [13]-[14]. In bilateral teleoperation research, a control system with acceleration measurement is designed in [15] using $\mathrm{NN}$ to estimate nonlinear uncertainties. In [16], the $\mathrm{NN}$ is applied in a Prescribed Performance Control (PPC) system. A terminal sliding mode control system with NN is also designed in [17]. These systems, however, perform only under extremely restricting assumptions that the time delay is constant and the external force is zero, both of which are against the reality of these systems. In [18] $\mathrm{NN}$ is deployed for systems with time-varying delays, but the approach requires precise knowledge of the external force as well as the coefficients of mass and damper of the external force. [19] and [20] extend the application of NN to multilateral teleoperation. The major drawbacks of these studies are some of the assumptions underlying them. For example, the positive constraints including disturbances are assumed to be restricted by large velocity signals, and the rate of time delays must be less than one. In [21], the state-of-art neural network control systems is reviewed.

The idea of passivity characterized by mechanical energy, which uses force and velocity as efforts and flow variables, is an effective tool for establishing stability of bilateral teleoperation interaction under time delays. Compared with the methods based on absolute stability, most passivity-based methods are more conservative and sacrifice system transparency, but can easily accommodate communication time delays. Among the numerous passivity-based approaches, the wave variable method, introduced by Niemeyer and Slotine, is a classic approach to guarantee the time delayed channel passivity. However, the traditional wave variable transformation has many drawbacks. For example, it can hardly guarantee the system stability when time delay varies. In addition, the two intrinsic problems in a wave variable system, position drift and wave reflections can cause inaccurate position tracking and large signal variations, respectively. Numerous approaches have been proposed to overcome one or some of these shortcomings [22]-[30]. Nevertheless, according to the literature, none of the previous work addresses all the problems associated with the wave 
variable transformation. Specially, the time-varying delay issue is still the main drawback of the previous work on wave-based systems.

Another classic passivity-based approach is Time Domain Passivity Approach (TDPA) that was first introduced by Hannaford et al., consisting of passivity observers and passivity controllers, to adaptively dissipate energy [31]. The passivity observers are used to monitor the channel passivity and the passivity controllers are used to dissipate the active energy. This method is later extended in [32] to deal with the time-varying delay issues. In [33] and [34], the energy-based TDPA is extended to the power-based TDPA, which can dissipates energy as soon as any active energy is produced. The power-based TDPA proposed in [34] is further extended in [35] to tackle the position drift issue. However, although the above TDPA-based system are capable to guarantee channels passivity under time-varying delays, transparency degradation is still their main drawback, especially in the presence of small constant or no time delay where high transparency can be easily derived by many nonpassivity based schemes. In [36], we proposed a wave-based TDPA system to more accurately observe the power flow during different time delay scenarios. However, assumption of the rate of time delays less than one and degraded position and torque tracking owning to passivity controllers in the presence of sharply-varying delays were two major weaknesses of that method.

In this paper, a new wave-based TDPA system is proposed to guarantee the communication channels' passivity and achieve high tracking performance in the presence of time-varying delays without rate restrictions. Compared with the previous powerbased TDPA, the proposed wave-based TDPA can more efficiently monitor the power flows under the condition of arbitrary time delays. The proposed passivity controllers do not influence position and force tracking. $\mathrm{NN}$ is applied to the proposed system to estimate and eliminate the dynamic uncertainties. The proposed control algorithm is deployed in the absence of the knowledge of the upper bound of the $\mathrm{NN}$ approximation error and external disturbance. Lyapunov functions are used to prove the system stability. Finally, the experimental work is performed to show the effectiveness of proposed control system in comparison with other systems in different scenarios.

The remainder of the paper is structured as follows: After providing a background in Section 2 on the dynamics of teleoperation system and its related properties, and the knowledge of the Radial Basis Function (RBF) NN, the proposed four-channel (4-CH) wave-based TDPA is described in Section 3. In Section 4, the NN-based controller design is introduced and the delay-based stability is also studied. Results of the experimental work are presented in Section 5. Section 6 draws some conclusions.

\section{Background}

\subsection{Model of a teleoperation system}

In this paper, the local (master) and the remote (slave) robots are modeled as a pair of n-DOF serial links with revolute joints. Their corresponding nonlinear dynamics are modelled as:

$M_{m}\left(q_{m}\right) \ddot{q}_{m}+C_{m}\left(q_{m}, \dot{q}_{m}\right) \dot{q}_{m}+F_{m} \dot{q}_{m}+f_{c m}\left(\dot{q}_{m}\right)+g_{m}\left(q_{m}\right)-$

$F_{m}^{*}=\tau_{m}+\tau_{h}$

$M_{s}\left(q_{s}\right) \ddot{q}_{s}+C_{s}\left(q_{s}, \dot{q}_{s}\right) \dot{q}_{s}+F_{s} \dot{q}_{s}+f_{c s}\left(\dot{q}_{s}\right)+g_{s}\left(q_{s}\right)-F_{s}^{*}=$

$\tau_{s}-\tau_{e}$ where $i=m, s$ for the master and slave. $\ddot{q}_{i}(t), \dot{q}_{i}(t), q_{i}(t) \in$ $R^{n \times 1}$ are the joint acceleration, velocity and position, respectively. $M_{i}\left(q_{i}(t)\right) \in R^{n \times n}$ are the inertia matrices, $C_{i}\left(q_{i}(t), \dot{q}_{i}(t)\right) \in R^{n \times n} \quad$ are Coriolis/centrifugal effects. $g_{i}\left(q_{i}(t)\right) \in R^{n}$ are the vectors of gravitational forces and $\tau_{i}$ are the control signals. $\tau_{h}(t)$ and $\tau_{e}(t)$ are the actual human and environmental torques applied to the robots. $F_{i} \dot{q}_{i}(t)$ denote the viscous friction and $f_{c i}\left(\dot{q}_{i}(t)\right)$ denote the Coulomb friction. $F_{i}^{*}(t) \in R^{n \times 1}$ are the bounded unknown disturbances. In the paper, the Coulomb friction function $f_{c i}\left(\dot{q}_{i}(t)\right)$ on the master and slave sides are bounded and piecewise continuous functions. Important properties of the above nonlinear dynamic model, which are used in this paper, are as follows:

P1: The inertia matrix $M_{i}\left(q_{i}\right)$ for a manipulator is symmetric positive-definite as: $0<\sigma_{\min }\left(M_{i}\left(q_{i}(t)\right)\right) I \leq M_{i}\left(q_{i}(t)\right) \leq$ $\sigma_{\max }\left(M_{i}\left(q_{i}(t)\right)\right) I \leq \infty$, where $\mathrm{I} \in \mathrm{R}^{\mathrm{n} \times \mathrm{n}}$ is the identity matrix. $\sigma_{\min }$ and $\sigma_{\max }$ denote the strictly positive minimum (maximum) eigenvalue of $M_{i}$ for all configurations $q_{i}$.

P2: Under an appropriate definition of the Coriolis/centrifugal matrix, the matrix $\dot{M}_{i}-2 C_{i}$ is skew symmetric, which can also be expressed as:

$\dot{M}_{i}\left(q_{m}(t)\right)=C_{i}\left(q_{i}(t), \dot{q}_{i}(t)\right)+C_{i}^{T}\left(q_{i}(t), \dot{q}_{i}(t)\right)$

P3: For a manipulator with revolute joints, there exists a positive constant $Z$ bounding the Coriolis/centrifugal matrix as:

$\left\|C_{i}\left(q_{i}(t), x(t)\right) y(t)\right\|_{2} \leq Z\|x(t)\|_{2}\|y(t)\|_{2}$

P4: The time derivative of $C_{i}\left(q_{i}(t), \dot{q}_{i}(t)\right)$ is bounded if $q_{i}(t)$ and $\dot{q}_{i}(t)$ are bounded.

In this paper, the external human and environmental torques are modelled as (5) and (6), where $\tau_{h, e}^{*}(t)$ stand for, respectively, the positive and bounded human operator and the environment exogenous input. $K_{h, e}, B_{h, e}$ and $M_{h, e}$ represent the nonnegative constant scalars corresponding to the mass, damping and stiffness of human and environment. $\Delta k_{h, e}, \Delta b_{h, e}, \Delta m_{h, e}$ are the unknown bounded variables relating to $K_{h, e}, B_{h, e}$ and $M_{h, e}$. Moreover, we use the extended active observer (EAOB) to measure the human and environmental torques as well as acceleration signals [26]. Compared with other force observers, EAOB possesses the advantage of external noise suppression by deploying Kalman filter, and is suitable for nonlinear systems.

$\tau_{h}(t)=\tau_{h}^{*}(t)-\left(K_{h}+\Delta k_{h}\right) q_{m}(t)-\left(B_{h}+\Delta b_{h}\right) \dot{q}_{m}(t)-$
$\left(M_{h}+\Delta m_{h}\right) \ddot{q}_{m}(t)$
$\tau_{e}(t)=\tau_{e}^{*}(t)+\left(K_{e}+\Delta k_{e}\right) q_{s}(t)+\left(B_{e}+\Delta b_{e}\right) \dot{q}_{s}(t)+\left(M_{e}+\right.$
$\left.\Delta m_{e}\right) \ddot{q}_{s}(t)$

2.2. Neural networks (NN)

The main advantage of the NNs is its ability to approximate any smooth nonlinear function with arbitrary precision due to its inherent approximate capabilities [15], [38]. In this paper, the Radial Basis Function (RBF) NN is applied to approximate a continuous function $f(X): R^{q} \rightarrow R^{p}$ expressed as:

$f(X)=W^{T} \Phi(X)+\xi(X)$

where $X \in \Omega_{x} \subset R^{q}$ is the input vector. $W \in R^{n \times p}$ is the weight matrix. $\mathrm{n}$ is the number of the neurons. $\xi(X)$ is the approximation errors.

$\Phi(X)=\left[\Phi_{1}(X), \Phi_{2}(X), \ldots, \Phi_{k}(X), \ldots, \Phi_{n}(X)\right]$, where $\Phi_{k}(X)$ is 
the RBF Gaussian function:

$$
\Phi_{k}(X)=\exp \left(-\frac{1}{2 \mathrm{H}_{k}{ }^{2}}\left\|X-C_{k}\right\|^{2}\right)
$$

Where $C_{k}$ and $\mathrm{H}_{k}$ are the center and the width of the k-th neuron, respectively. According to the universal approximation property of NNs, for any continuous function $\mathrm{f}(\mathrm{X})$, there exists an NN such that

$f(X)=W^{* T} \Phi(X)+\xi^{*}(X),\left\|\xi^{*}(X)\right\| \leq \xi_{u p}^{*}$

where $W^{*}$ and $\xi^{*}(X)$ are the ideal weight and error in the approximation, respectively. $\xi_{u p}^{*}$ is $\xi^{*}(X)$ 's upper bound. The dynamic functions of the manipulaters can be considered to be piecewise continuous functions due to the existing friction and backlash. Assume that $f(X)$ is a piecewise function which can be written as: $f(X)=f_{1}(X)+f_{2}(X)$, where $f_{1}(X)$ is the continuous part and $f_{2}(X)$ is the bounded piecewise term, respectively. Therefore:

$f(X)=W^{* T} \Phi(X)+\xi^{*}(X)+f_{2}(X)=W^{* T} \Phi(X)+\bar{\xi}^{*}(X)$

where $\bar{\xi}^{*}(X)=\xi^{*}(X)+f_{2}(X), \bar{\xi}^{*}(X) \leq \bar{\xi}_{u p}^{*} . \bar{\xi}_{u p}^{*}$ is the upper bound of the approximation error.

\section{Wave-based TDPA}

The system passivity in a traditional power-based TDPA system can be defined as [34], [35]:

$$
\begin{aligned}
& P(t)=\tau_{m}(t) \dot{q}_{m}(t)-\tau_{s}(t) \dot{q}_{s}(t)=\frac{1}{2 b} \tau_{m}^{T}(t) \tau_{m}(t)- \\
& \frac{1}{2 b}\left(\tau_{m}(t)-b \dot{q}_{m}(t)\right)^{2}+b \dot{q}_{s}^{T}(t) \dot{q}_{s}(t)-\frac{1}{2 b}\left(\tau_{s}(t)+b \dot{q}_{s}(t)\right)^{2}- \\
& \frac{1}{2 b} \dot{T}_{2}(t) \tau_{m}^{T}(t) \tau_{m}(t)-\frac{b}{2} \dot{T}_{1}(t) \dot{q}_{s}^{T}(t) \dot{q}_{s}(t)+\frac{d E}{d t}=P_{\text {diss }}+\frac{d E}{d t} \\
& E(t)=\frac{1}{2 b} \int_{t-T_{2}(t)}^{t} \tau_{s}^{T}(\eta) \tau_{s}(\eta) d \eta+\frac{b}{2} \int_{t-T_{1}(t)}^{t} \dot{q}_{m}^{T}(\eta) \dot{q}_{m}(\eta) d \eta(12)
\end{aligned}
$$

where $\mathrm{b}$ is a positive constant that relates to the unit of torque and velocity. $T_{1}(t)$ and $T_{2}(t)$ are the forward and backward time delays, respectively. Since $P_{\text {diss }}$ is not observable at any single port of the 2-port network, in order to facilitate real-time monitoring of the network's passivity, $P_{\text {diss }}$ can be written as:

$P_{\text {diss }}(t)=P_{\text {diss }}^{m}(t)+P_{\text {diss }}^{s}(t)$

where $P_{\text {diss }}^{m}(t)$ and $P_{\text {diss }}^{S}(t)$ are the power dissipation components which are observable at the master and slave ports, respectively.

$P_{\text {diss }}^{m}(t)=\frac{1}{b} \tau_{m}^{T}(t) \tau_{m}(t)-\frac{1}{2 b}\left(\tau_{m}(t)-b \dot{q}_{m}(t)\right)^{2}-$

$\frac{1}{2 b} \dot{T}_{2}(t) \tau_{m}^{T}(t) \tau_{m}(t)$

$P_{\text {diss }}^{S}(t)=b \dot{q}_{s}^{T}(t) \dot{q}_{s}(t)-\frac{1}{2 b}\left(\tau_{s}(t)+b \dot{q}_{s}(t)\right)^{2}-$

$\frac{b}{2} \dot{T}_{1}(t) \dot{q}_{s}^{T}(t) \dot{q}_{s}(t)$

$\dot{T}_{1,2}$ is replaced by constant parameters $\bar{\mu}_{1,2}$ in [34]-[35]. Their values are set to be the upper bound of $\dot{T}_{1,2}$. The passivity observers on the master and the slave side can be written as:

$P_{o b s}^{m}(t)=\frac{1}{b} \tau_{m}^{T}(t) \tau_{m}(t)-\frac{1}{2 b}\left(\tau_{m}(t)-b \dot{q}_{m}(t)\right)^{2}-$

$\frac{1}{2 b} \bar{\mu}_{2} \tau_{m}^{T}(t) \tau_{m}(t)$

$P_{o b s}^{s}(t)=b \dot{q}_{s}^{T}(t) \dot{q}_{s}(t)-\frac{1}{2 b}\left(\tau_{s}(t)+b \dot{q}_{s}(t)\right)^{2}-$

$\frac{b}{2} \bar{\mu}_{1} \dot{q}_{s}^{T}(t) \dot{q}_{s}(t)$
By applying the passivity observers, the power flows can be detected in each port. Two passivity controllers attached at each port are activated when $P_{o b s}^{m}$ and $P_{o b s}^{s}$ are negative so that $P_{c t r}^{m}=-P_{o b s}^{m}$ and $P_{c t r}^{s}=-P_{o b s}^{s}$ where $P_{c t r}^{m}$ and $P_{c t r}^{s}$ are the dissipated power from the passivity controllers. By using the two passivity controllers, the torque perceived by the operator $\tau_{m}^{\prime}(t)$ and the command velocity of slave $\dot{q}_{s}^{\prime}(t)$ can be derived as [34]:

$$
\begin{aligned}
\tau_{m}^{\prime}(t) & =\tau_{s}\left(t-T_{2}(t)\right)+\tau_{P C}(t) \\
\dot{q}_{s}^{\prime}(t) & =\dot{q}_{m}\left(t-T_{1}(t)\right)-\dot{q}_{P C}(t)
\end{aligned}
$$

where $\tau_{P C}(t)$ is the output of the master side passivity controller and $\dot{q}_{P C}(t)$ is the output of the slave side passivity controller. The power-based TDPA using the passivity observers and passivity controllers can robustly guarantee the passivity of the communication channels in the presence of time varying delays. However, as a conservative method for system passivity, this method can largely degrade the system's transparency in the presence of the constant time delays or even no delay $\left(\bar{\mu}_{2}=0\right)$. During the free space movement $\left(\tau_{m, s}=0\right)$, (16) can be simplified as $P_{o b s}^{m}(t)=-\frac{b}{2} \dot{q}_{m}^{2}(t)$, and during the hard environmental contact $\left(\dot{\mathrm{q}}_{\mathrm{m}, \mathrm{s}}=0\right)$, (17) can be simplified as $\mathrm{P}_{\mathrm{obs}}^{\mathrm{s}}(\mathrm{t})=-\frac{1}{2 \mathrm{~b}} \tau_{\mathrm{s}}^{2}(\mathrm{t})$. Under these conditions, $P_{o b s}^{m}(t)$ and $P_{o b s}^{s}(t)$ are negative to the extent that accurate torque and trajectory tracking performances cannot be achieved due to the adverse effect of the passivity controllers.

Remark. In this paper, the differentials of unsymmetrical time delays $T_{1}(t)$ and $T_{2}(t)$ are bounded by $\bar{\mu}_{1,2}$. That is, $\left|\dot{T}_{1,2}(t)\right| \leq \bar{\mu}_{1,2} \cdot \bar{\mu}_{1,2} \quad$ are arbitrary positive constants. Moreover, the time-varying delays $T_{1,2}(t)$ are considered to be the sum of the constant time delays $\bar{T}_{1,2}$ with their bounded perturbations $\Delta T_{1,2}(t)$. That is, $T_{1,2}(t)=\bar{T}_{1,2}+\Delta T_{1,2}(t) \leq$ $\bar{T}_{1,2}+\bar{\varepsilon}_{1,2}=T_{1,2}^{\max }$, where $\bar{\varepsilon}_{1,2}$ are the upper bounds of the perturbations and $T_{1,2}^{\max }$ are the upper bounds of the $T_{1,2}(t)$.

Fig.1 shows the proposed 4-CH wave variable transformation which contains two wave transformation schemes.

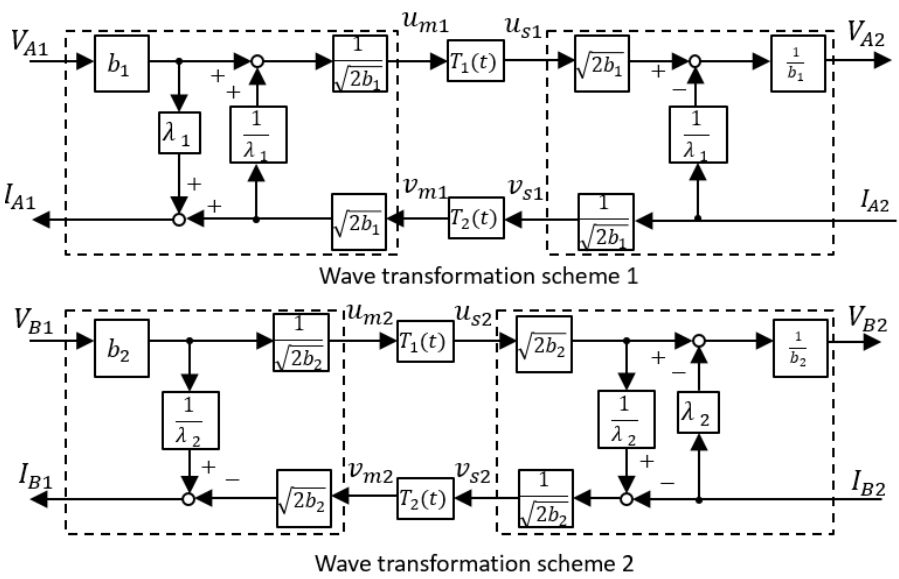

Fig.1. 4-CH wave variable transformation

The two wave transformation schemes are applied to encode the feed-forward signals $V_{A 1}$ and $V_{B 1}$ with the feedback signals $I_{A 2}$ and $I_{B 2}$, where $V_{A 1}(t)=\beta \delta q_{m}(t)+\alpha \delta \dot{q}_{m}(t)+\gamma \delta \ddot{q}_{m}(t)$, $V_{B 1}(t)=\alpha_{1} \delta \dot{q}_{m}(t)+\gamma_{1} \delta \ddot{q}_{m}(t), I_{A 2}(t)=\alpha_{1} \delta \dot{q}_{s}(t)+$ $+\gamma_{1} \delta \ddot{q}_{m}(t), I_{B 2}(t)=-\beta \delta q_{s}(t)-\alpha \delta \dot{q}_{s}(t)-\gamma \delta \ddot{q}_{s}(t) . \alpha, \alpha_{1}$, $\gamma, \gamma_{1}$ and $\beta$, are diagonal positive-definite matrices. $\delta$ is a positive constant. In this system, position, velocity and 
acceleration are transmitted between the two robots.

The wave variables in the two schemes are defined as follows:

$$
\begin{aligned}
& u_{m 1}(t)=\frac{b_{1} V_{A 1}(t)+\frac{1}{\lambda_{1}} I_{A 2}\left(t-T_{2}(t)\right)}{\sqrt{2 b_{1}}}, u_{s 1}(t)=\frac{b_{1} V_{A 2}(t)+\frac{1}{\lambda_{1}} I_{A 2}(t)}{\sqrt{2 b_{1}}} \\
& v_{m 1}(t)=\frac{I_{A 2}\left(t-T_{2}(t)\right)}{\sqrt{2 b_{1}}}, v_{s 1}(t)=\frac{I_{A 2}(t)}{\sqrt{2 b_{1}}} \\
& u_{m 2}(t)=\frac{b_{2} V_{B 1}(t)}{\sqrt{2 b_{2}}}, u_{s 2}(t)=\frac{b_{2} V_{B 1}\left(t-T_{1}(t)\right)}{\sqrt{2 b_{2}}} \\
& v_{m 2}(t)=\frac{\frac{b_{2}}{\lambda_{2}} V_{B 1}(t)-I_{B 1}(t)}{\sqrt{2 b_{2}}}, v_{s 2}(t)=\frac{\frac{b_{2}}{\lambda_{2}} V_{B 1}\left(t-T_{1}(t)\right)-I_{B 2}(t)}{\sqrt{2 b_{2}}}
\end{aligned}
$$

where $b_{1,2}$ and $\lambda_{1,2}$ are the positive characteristic impedances. The traditional wave variable transformation is written as $u_{m}(t)=-v_{m}(t)+\sqrt{2 b} \dot{q}_{m}(t), v_{s}(t)=-u_{s}(t)+\sqrt{2 / b} F_{e}(t)$ in [11], where the incoming wave variables $v_{m}$ and $u_{s}$ in this relationship are reflected and returned as the outgoing wave variable $u_{m}$ and $v_{s}$. This phenomenon is called wave reflection. Wave reflections can last several cycles in the communication channels and then gradually disappear, which can easily produce unpredictable interference and disturbances that significantly influence transparency. Unlike the conventional wave variable, the outgoing wave variables $v_{\mathrm{s} 1}$ and $\mathrm{u}_{\mathrm{m} 2}$ do not contain any unnecessary information from the incoming wave variables $u_{s 1}$ and $v_{m 2}$ as shown in (21)-(22). Therefore, the signal variations caused by wave-reflections can be efficiently reduced. In addition, direct position information is transmitted between the master and the slave, and position drift does not occur in this system. The control signals after transmission in Fig. 1 can be derived as

$I_{A 1}=\alpha_{1} \delta \dot{q}_{s}\left(t-T_{2}(t)\right)+\gamma_{1} \delta \ddot{q}_{s}\left(t-T_{2}(t)\right)+$

$b_{1} \lambda_{1}\left(\beta \delta q_{m}(t)+\alpha \delta \dot{q}_{m}(t)+\gamma \delta \ddot{q}_{m}(t)\right)$

$I_{B 1}=-\beta \delta q_{s}\left(t-T_{2}(t)\right)-\alpha \delta \dot{q}_{s}\left(t-T_{2}(t)\right)-\gamma \delta \ddot{q}_{s}(t-$

$\left.T_{2}(t)\right)+\frac{b_{2} \alpha_{1}}{\lambda_{2}}\left(\delta \dot{q}_{m}(t)-\delta \dot{q}_{m}\left(t-T_{1}(t)-T_{2}\left(t-T_{1}(t)\right)\right)\right)$

$V_{A 2}(t)=\beta \delta q_{m}\left(t-T_{1}(t)\right)+\alpha \delta \dot{q}_{m}\left(t-T_{1}(t)\right)+\gamma \delta \ddot{q}_{m}(t-$

$\left.T_{1}(t)\right)-\frac{\alpha_{1}}{b_{1} \lambda_{1}}\left(\delta \dot{q}_{s}(t)-\delta \dot{q}_{s}\left(t-T_{2}(t)-T_{1}\left(t-T_{2}(t)\right)\right)\right)$

$V_{B 2}(t)=\alpha_{1} \delta \dot{q}_{m}\left(t-T_{1}(t)\right)+\gamma_{1} \delta \ddot{q}_{m}\left(t-T_{1}(t)\right)+$

$\frac{\lambda_{2}}{b_{2}}\left(\beta \delta q_{s}(t)+\alpha \delta \dot{q}_{s}(t)+\gamma \delta \ddot{q}_{s}(t)\right)$

In order to simplify this expression, we define $T_{l 1}(t)=T_{2}(t)+$ $T_{1}\left(t-T_{2}(t)\right)$ and $T_{l 2}(t)=T_{1}(t)+T_{2}\left(t-T_{1}(t)\right)$. We also have the constraints $\dot{T}_{l 1}(t) \leq \bar{\mu}_{1}, \dot{T}_{l 2}(t) \leq \bar{\mu}_{2}$. The proposed 4$\mathrm{CH}$ wave variable transformation can actually be seen as the combination of two 2-port networks. Therefore, the power flow in the 4-CH wave variable transformation can be defined as:

$$
P_{4 C H}(t)=P_{1}(t)[\text { scheme } 1]+P_{2}(t)[\text { scheme } 2]
$$

$$
\begin{aligned}
& P_{1}(t)=V_{A 1}(t) I_{A 1}(t)-V_{A 2}(t) I_{A 2}(t) \\
& =2 \lambda_{1} u_{m 1}^{T}(t) u_{m 1}(t)+\frac{2}{\lambda_{1}} v_{s 1}^{T}(t) v_{s 1}(t)-2\left(u_{m 1}^{T}(t) v_{m 1}(t)+\right. \\
& \left.u_{s 1}^{T}(t) v_{s 1}(t)\right) \\
& =\lambda_{1}\left(u_{m 1}^{T}(t) u_{m 1}(t)-u_{s 1}^{T}(t) u_{s 1}(t)\right)+\frac{1}{\lambda_{1}}\left(v_{s 1}^{T}(t) v_{s 1}(t)-\right. \\
& \left.v_{m 1}^{T}(t) v_{m 1}(t)\right)+\frac{1}{\lambda_{1}}\left(v_{m 1}(t)-\lambda_{1} u_{m 1}(t)\right)^{T}\left(v_{m 1}(t)-\right.
\end{aligned}
$$

$$
\begin{aligned}
& \left.\lambda_{1} u_{m 1}(t)\right)+\frac{1}{\lambda_{1}}\left(v_{s 1}(t)-\lambda_{1} u_{s 1}(t)\right)^{T}\left(v_{s 1}(t)-\lambda_{1} u_{s 1}(t)\right) \\
& =\frac{d}{d t} \int_{t-T_{1}(t)}^{t} \lambda_{1} u_{m 1}^{T}(\eta) u_{m 1}(\eta) d \eta+ \\
& \frac{d}{d t} \int_{t-T_{2}(t)}^{t} \frac{1}{\lambda_{1}} v_{s 1}^{T}(\eta) v_{s 1}(\eta) d \eta+\frac{1}{\lambda_{1}}\left(v_{m 1}(t)-\right. \\
& \left.\lambda_{1} u_{m 1}(t)\right)^{T}\left(v_{m 1}(t)-\lambda_{1} u_{m 1}(t)\right)+\frac{1}{\lambda_{1}}\left(v_{s 1}(t)-\right. \\
& \left.\lambda_{1} u_{s 1}(t)\right)^{T}\left(v_{s 1}(t)-\lambda_{1} u_{s 1}(t)\right)-\lambda_{1} \dot{T}_{1}(t) u_{s 1}^{T}(t) u_{s 1}(t)- \\
& \frac{1}{\lambda_{1}} \dot{T}_{2}(t) v_{m 1}^{T}(t) v_{m 1}(t) \\
& =\frac{d E_{1}(t)}{d t}+P_{1}^{d i s s}(t) \\
& E_{1}(t)=\int_{t-T_{1}(t)}^{t} \lambda_{1} u_{m 1}^{T}(\eta) u_{m 1}(\eta) d t+ \\
& \int_{t-T_{2}(t)}^{t} \frac{1}{\lambda_{1}} v_{s 1}^{T}(\eta) v_{s 1}(\eta) d t
\end{aligned}
$$

$P_{1}^{\text {diss }}(t)=\frac{1}{\lambda_{1}}\left(v_{m 1}(t)-\lambda_{1} u_{m 1}(t)\right)^{T}\left(v_{m 1}(t)-\lambda_{1} u_{m 1}(t)\right)+$ $\frac{1}{\lambda_{1}}\left(v_{s 1}(t)-\lambda_{1} u_{s 1}(t)\right)^{T}\left(v_{s 1}(t)-\lambda_{1} u_{s 1}(t)\right)-$$$
\lambda_{1} \dot{T}_{1}(t) u_{s 1}^{T}(t) u_{s 1}(t)-\frac{1}{\lambda_{1}} \dot{T}_{2}(t) v_{m 1}^{T}(t) v_{m 1}(t)
$$

$P_{2}(t)=V_{B 1}(t) I_{B 1}(t)-V_{B 2}(t) I_{B 2}(t)$

$=\frac{2}{\lambda_{2}} u_{m 2}^{T}(t) u_{m 2}(t)+2 \lambda_{2} v_{s 2}^{T}(t) v_{s 2}(t)-2\left(u_{m 2}^{T}(t) v_{m 2}(t)+\right.$ $\left.u_{s 2}^{T}(t) v_{s 2}(t)\right)$

$=\frac{1}{\lambda_{2}}\left(u_{m 2}^{T}(t) u_{m 2}(t)-u_{s 2}^{T}(t) u_{s 2}(t)\right)+\lambda_{2}\left(v_{s 2}^{T}(t) v_{s 2}(t)-\right.$

$\left.v_{m 2}^{T}(t) v_{m 2}(t)\right)+\lambda_{2}\left(v_{m 2}(t)-\frac{1}{\lambda_{2}} u_{m 2}(t)\right)^{T}\left(v_{m 2}(t)-\right.$

$\left.\frac{1}{\lambda_{2}} u_{m 2}(t)\right)+\lambda_{2}\left(v_{s 2}(t)-\frac{1}{\lambda_{2}} u_{s 2}(t)\right)^{T}\left(v_{s 2}(t)-\frac{1}{\lambda_{2}} u_{s 2}(t)\right)$

$=\frac{d}{d t} \int_{t-T_{1}(t)}^{t} \frac{1}{\lambda_{2}} u_{m 2}^{T}(\eta) u_{m 2}(\eta) d \eta+$

$\frac{d}{d t} \int_{t-T_{2}(t)}^{t} \lambda_{2} v_{s 2}^{T}(\eta) v_{s 2}(\eta) d \eta+\lambda_{2}\left(v_{m 2}(t)-\right.$

$\left.\frac{1}{\lambda_{2}} u_{m 2}(t)\right)^{T}\left(v_{m 2}(t)-\frac{1}{\lambda_{2}} u_{m 2}(t)\right)+\lambda_{2}\left(v_{s 2}(t)-\right.$

$\left.\frac{1}{\lambda_{2}} u_{s 2}(t)\right)^{T}\left(v_{s 2}(t)-\frac{1}{\lambda_{2}} u_{s 2}(t)\right)-\frac{1}{\lambda_{2}} \dot{T}_{1}(t) u_{s 2}^{T}(t) u_{s 2}(t)-$

$\lambda_{2} \dot{T}_{2}(t) v_{m 2}^{T}(t) v_{m 2}(t)$

$=\frac{d E_{2}(t)}{d t}+P_{2}^{\text {diss }}(t)$

$E_{2}(t)=\int_{t-T_{1}(t)}^{t} \frac{1}{\lambda_{2}} u_{m 2}^{T}(\eta) u_{m 2}(\eta) d \eta+$

$\int_{t-T_{2}(t)}^{t} \lambda_{2} v_{s 2}^{T}(\eta) v_{s 2}(\eta) d \eta$

$P_{2}^{\text {diss }}(t)=\lambda_{2}\left(v_{m 2}(t)-\frac{1}{\lambda_{2}} u_{m 2}(t)\right)^{T}\left(v_{m 2}(t)-\frac{1}{\lambda_{2}} u_{m 2}(t)\right)+$

$\lambda_{2}\left(v_{s 2}(t)-\frac{1}{\lambda_{2}} u_{s 2}(t)\right)^{T}\left(v_{s 2}(t)-\frac{1}{\lambda_{2}} u_{s 2}(t)\right)-$

$\frac{1}{\lambda_{2}} \dot{T}_{1}(t) u_{s 2}^{T}(t) u_{s 2}(t)-\lambda_{2} \dot{T}_{2}(t) v_{m 2}^{T}(t) v_{m 2}(t)$

According to (29) and (32), the net energy flows are absolutely positive to guarantee passivity of the communication network. Based on the definition of passivity and assuming $E_{1}(0)=$ $E_{2}(0)=0$, the energy flow is derived as:

$E_{\text {flow }}(t)=\int_{0}^{t} P_{4 C H}(\eta) d \eta=\int_{0}^{t}\left(P_{1}^{\text {diss }}(\eta)+P_{2}^{\text {diss }}(\eta)+\right.$ $\left.\frac{d E_{1}}{d t}(\eta)+\frac{d E_{2}}{d t}(\eta)\right) d \eta=E_{1}(t)+E_{2}(t)-E_{1}(0)-E_{2}(0)+$ $\int_{0}^{t} P_{1}^{\text {diss }}(\eta)+P_{2}^{\text {diss }}(\eta) d \eta \geq \int_{0}^{t} P_{1}^{\text {diss }}(\eta)+P_{2}^{\text {diss }}(\eta) d \eta$ 
Therefore, in the situation that $P_{1}^{\text {diss }}(\mathrm{t})+P_{2}^{\text {diss }}(\mathrm{t}) \geq 0$, according to (34), the energy flow $E_{\text {flow }}(t)$ is no less than zero and the passivity of the time delayed network can be guaranteed. Similar with (11), $P_{1}^{\text {diss }}(\mathrm{t})+P_{2}^{\text {diss }}(\mathrm{t})$ can also be defined as the sum of master power dissipation components $P_{\text {diss }}^{m}(t)$ and slave power dissipation components $P_{\text {diss }}^{S}(t)$ based on (30) and (33).

$\mathrm{P}_{\mathrm{diss}}^{\mathrm{m}}(\mathrm{t})=\frac{1}{\lambda_{1}}\left(v_{m 1}(t)-\lambda_{1} u_{m 1}(t)\right)^{T}\left(v_{m 1}(t)-\lambda_{1} u_{m 1}(t)\right)+$

$\lambda_{2}\left(v_{m 2}(t)-\frac{1}{\lambda_{2}} u_{m 2}(t)\right)^{T}\left(v_{m 2}(t)-\frac{1}{\lambda_{2}} u_{m 2}(t)\right)-$

$\frac{1}{\lambda_{1}} \dot{T}_{2}(t) v_{m 1}^{T}(t) v_{m 1}(t)-\lambda_{2} \dot{T}_{2}(t) v_{m 2}^{T}(t) v_{m 2}(t)$

$\mathrm{P}_{\text {diss }}^{\mathrm{s}}(\mathrm{t})=\frac{1}{\lambda_{1}}\left(v_{s 1}(t)-\lambda_{1} u_{s 1}(t)\right)^{T}\left(v_{s 1}(t)-\lambda_{1} u_{s 1}(t)\right)+$

$\lambda_{2}\left(v_{s 2}(t)-\frac{1}{\lambda_{2}} u_{s 2}(t)\right)^{T}\left(v_{s 2}(t)-\frac{1}{\lambda_{2}} u_{s 2}(t)\right)-$

$\lambda_{1} \dot{T}_{1}(t) u_{s 1}^{T}(t) u_{s 1}(t)-\frac{1}{\lambda_{2}} \dot{T}_{1}(t) u_{s 2}^{T}(t) u_{s 2}(t)$

According to (35) and (36), the proposed passivity observers can observe the power dissipation components in real time, as $\mathrm{P}_{\text {diss }}^{\mathrm{m}}(\mathrm{t})$ and $\mathrm{P}_{\text {diss }}^{\mathrm{s}}(\mathrm{t})$ only contain the signals observed at the master and slave ports, respectively. The proposed 4-CH wave transformation is proposed to guarantee the passivity of the communication channels in the presence of constant delays so that the $P_{\text {diss }}^{m}(t)$ and $P_{\text {diss }}^{s}(t)$ are required to be positive when $\dot{T}_{1}(t)=\dot{T}_{2}(t)=0$. Therefore, for constant time delays, the final two terms in (35) and (36) can be treated as zero and then (35) and (36) are definitely non-negative. Therefore, the communication channels' passivity can be guaranteed by the proposed 4-CH wave transformation and the passivity controllers will not be launched to degrade the system transparency.

The value of $\dot{T}_{1,2}$ can be measured by using the time delay differential estimator in Fig.2. When this estimator is used, the integral of $\mathrm{u}_{\mathrm{m} 1}(\mathrm{t})$ and $\mathrm{v}_{\mathrm{s} 1}(\mathrm{t})$ should be sent outside the wave transformation. The passivity observers are designed as:

$P_{o b s}^{m}(t)=\frac{1}{\lambda_{1}}\left(v_{m 1}(t)-\lambda_{1} u_{m 1}(t)\right)^{T}\left(v_{m 1}(t)-\lambda_{1} u_{m 1}(t)\right)+$

$\lambda_{2}\left(v_{m 2}(t)-\frac{1}{\lambda_{2}} u_{m 2}(t)\right)^{T}\left(v_{m 2}(t)-\frac{1}{\lambda_{2}} u_{m 2}(t)\right)-$

$\frac{\hat{\hat{T}}_{2}}{\lambda_{1}} v_{m 1}^{T}(t) v_{m 1}(t)-\lambda_{2} \hat{\dot{T}}_{2} v_{m 2}^{T}(t) v_{m 2}(t)$

$P_{o b s}^{s}(t)=\frac{1}{\lambda_{1}}\left(v_{s 1}(t)-\lambda_{1} u_{s 1}(t)\right)^{T}\left(v_{s 1}(t)-\lambda_{1} u_{s 1}(t)\right)+$

$\lambda_{2}\left(v_{s 2}(t)-\frac{1}{\lambda_{2}} u_{s 2}(t)\right)^{T}\left(v_{s 2}(t)-\frac{1}{\lambda_{2}} u_{s 2}(t)\right)-$

$\lambda_{1} \hat{\dot{T}}_{1} u_{s 1}^{T}(t) u_{s 1}(t)-\frac{\hat{T}_{1}}{\lambda_{2}} u_{s 2}^{T}(t) u_{s 2}(t)$

$\hat{\dot{T}}_{1,2}=$

$\left\{\begin{array}{c}\dot{T}_{1,2}^{\text {estimate }}, \text { if } \dot{T}_{1,2}^{\text {estimate }}<\overline{\mathrm{M}}_{1,2} \\ \bar{M}_{1,2} \text {, else, if } \dot{T}_{1,2}^{\text {estimate }} \geq \bar{M}_{1,2} \\ \bar{M}_{1,2}, \text { else, if } u_{m 1}\left(t-T_{1}(t)\right)=0 \text { or } v_{s 1}\left(t-T_{1}(t)\right)=0\end{array}\right.$

where $\bar{M}_{1,2}$ are the estimated constant upper bound that satisfy $\bar{M}_{1,2}>\dot{T}_{1,2}$.

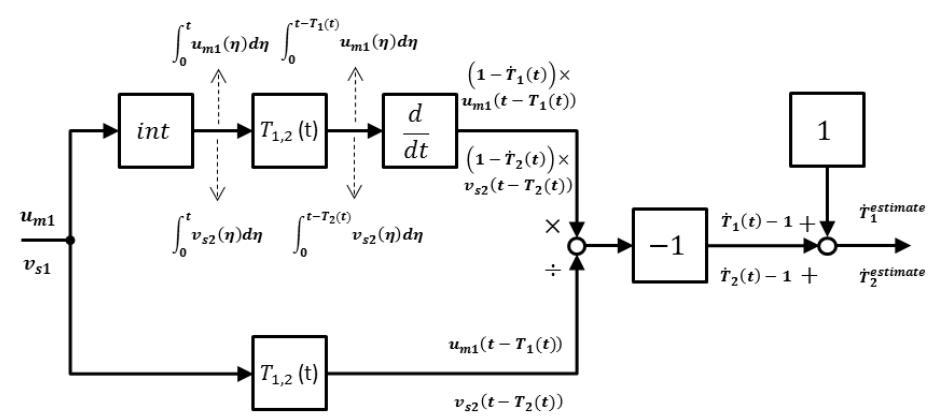

Fig.2. Time delay differential estimator

By using the passivity observer, we design the passivity controller to be:

$\widehat{V}_{s}(t)=V_{A 2}(t)-V_{B 2}(t)-\Gamma_{2}(t)$

$\hat{I}_{m}(t)=-I_{A 1}-I_{B 1}-\Gamma_{1}(t)$

where $\widehat{V}_{s}(t)$ and $\hat{I}_{m}(t)$ are the output control signals from the passivity controllers on the slave and master sides, respectively. $\Gamma_{1}(t)$ and $\Gamma_{2}(t)$ are designed as (42)-(43), where $\sigma_{1,2}, \varrho$ are positive constants.

$$
\begin{aligned}
& \Gamma_{1}(t)= \\
& \left\{\begin{array}{c}
0, \text { if } P_{o b s}^{m}(t) \geq 0 \\
\frac{\left(\alpha-\alpha_{1}\right) \bar{\mu}_{2} \delta \dot{q}_{s}^{T}\left(t-T_{2}(t)\right) \dot{q}_{s}\left(t-T_{2}(t)\right)}{2\left(\dot{q}_{m}(t)+e^{-\varrho t}\right)}+\frac{\left(\gamma-\gamma_{1}\right) \bar{\mu}_{2} \delta \ddot{q}_{S}^{T}\left(t-T_{2}(t)\right) \ddot{q}_{s}\left(t-T_{2}(t)\right)}{2\left(\dot{q}_{m}(t)+e^{-\varrho t}\right)} \\
+\frac{b_{2} \alpha_{1} \bar{\mu}_{2} \delta\left(\dot{q}_{m}^{T}(t) \dot{q}_{m}(t)+\sigma_{1}\right)}{2 \lambda_{2}\left(\dot{q}_{m}(t)+e^{-\varrho t}\right)} \text { if } P_{o b s}^{m}(t)<0
\end{array}\right.
\end{aligned}
$$

$$
\begin{aligned}
& \Gamma_{2}(t)= \\
& \left\{\begin{array}{c}
0, \quad \text { if } P_{o b s}^{s}(t) \geq 0 \\
\frac{\left(\alpha-\alpha_{1}\right) \bar{\mu}_{1} \delta \dot{q}_{m}^{T}\left(t-T_{1}(t)\right) \dot{q}_{m}\left(t-T_{1}(t)\right)}{2\left(\dot{q}_{s}(t)+e^{-\varrho t}\right)}+\frac{\left(\gamma-\gamma_{1}\right) \bar{\mu}_{1} \delta \ddot{q}_{m}^{T}\left(t-T_{1}(t)\right) \ddot{q}_{m}\left(t-T_{1}(t)\right)}{2\left(\dot{q}_{s}(t)+e^{-\varrho t}\right)} \\
+\frac{\alpha_{1} \bar{\mu}_{1} \sigma_{2} \delta\left(\dot{q}_{S}^{T}(t) \dot{q}_{s}(t)+\sigma_{2}\right)}{2 b_{1} \lambda_{1}\left(\dot{q}_{s}(t)+e^{-\varrho t}\right)}, \text { if } P_{o b s}^{S}(t)<0
\end{array}\right.
\end{aligned}
$$

\section{Design and analysis of the proposed teleoperation system}

Based on the external force models (5)-(6), the teleoperation dynamics can be rewritten as the following form:

$M_{m}\left(q_{m}\right) \delta \ddot{q}_{m}+C_{m}\left(q_{m}, \dot{q}_{m}\right) \delta \dot{q}_{m}=\tau_{m}+\tau_{h}^{*}(t)-B_{h} \delta \dot{q}_{m}(t)-$ $M_{h} \delta \ddot{q}_{m}(t)+F_{m}^{*}-f_{m}\left(X_{m}\right)$

$M_{s}\left(q_{s}\right) \delta \ddot{q}_{s}+C_{s}\left(q_{s}, \dot{q}_{s}\right) \delta \dot{q}_{s}=\tau_{s}-\tau_{e}^{*}(t)-B_{e} \delta \dot{q}_{s}(t)-$ $M_{e} \delta \ddot{q}_{s}(t)+F_{s}^{*}-f_{s}\left(X_{s}\right)$

where $X_{i}(t)=\left[\ddot{q}_{i}^{T}(t), \dot{q}_{i}^{T}(t), q_{i}^{T}(t)\right]^{T} . f_{i}\left(X_{i}\right)$ are defined as:

$$
\begin{aligned}
& f_{i}\left(X_{i}\right)=F_{i} \dot{q}_{i}+f_{c i}\left(\dot{q}_{i}\right)+g_{i}\left(q_{i}\right)+M_{i}\left(q_{i}\right)(1-\delta) \ddot{q}_{i}+ \\
& C_{i}\left(q_{i}, \dot{q}_{i}\right)(1-\delta) \dot{q}_{i}+\left(K_{h, e}+\Delta k_{h, e}\right) q_{i}+\Delta b_{h, e} \dot{q}_{i}+\Delta m_{h, e} \ddot{q}_{i}
\end{aligned}
$$

According to NNs approximation property, the functions $\hat{f}_{i}\left(X_{i}\right)$ are applied in this paper to approximate $f_{i}\left(X_{i}\right)$ with

$$
\hat{f}_{i}\left(X_{i}\right)=\widehat{W}_{i}^{T} \Phi_{i}\left(X_{i}\right)
$$




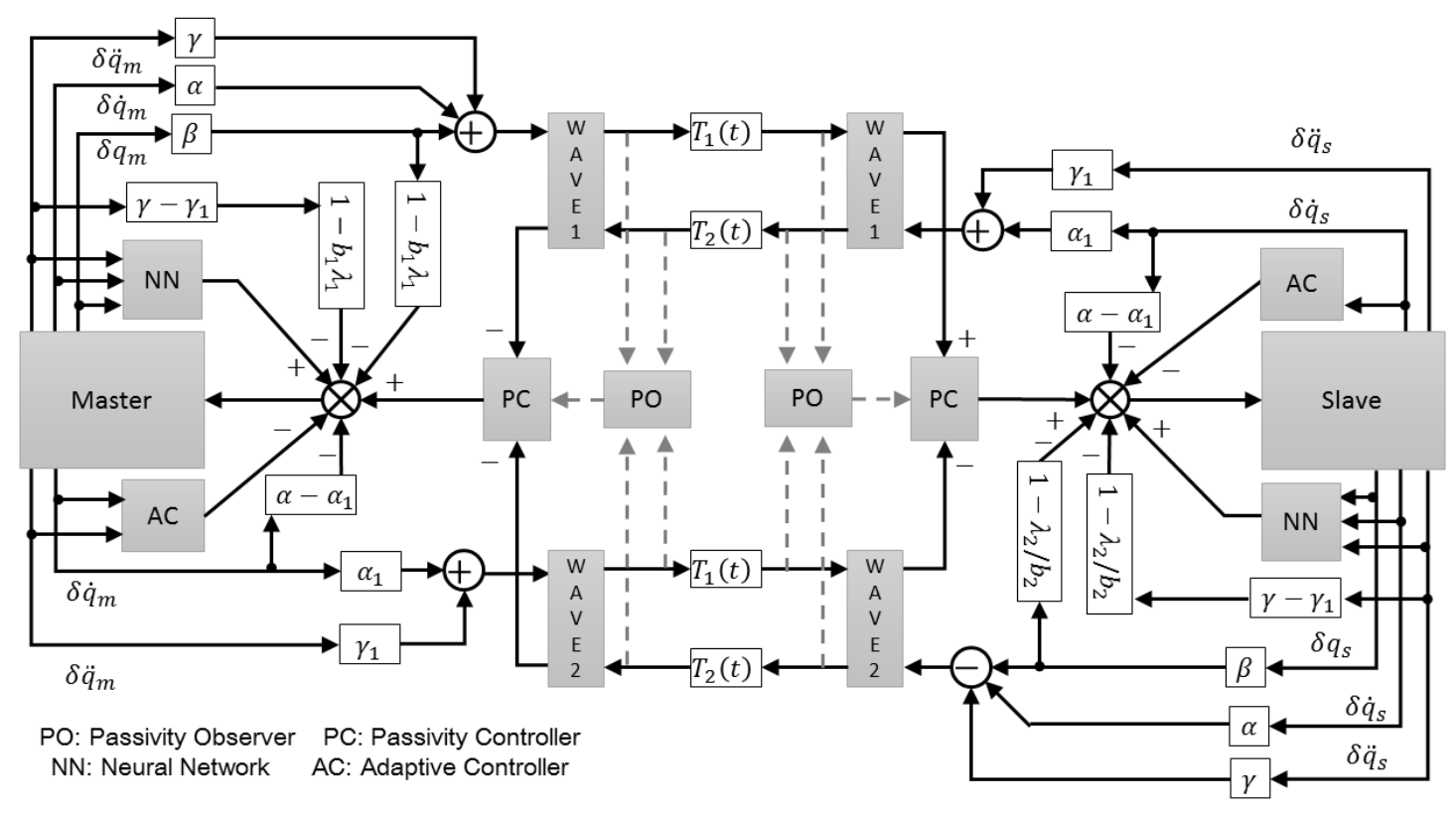

Fig.3. Total block diagram

where $\widehat{W}_{i}$ are the NN adaption parameters and $\Phi_{i}\left(X_{i}\right)$ are the $\mathrm{NN}$ basis functions. We define

$\tilde{f}_{i}\left(X_{i}\right)=\hat{f}_{i}\left(X_{i}\right)-f_{i}\left(X_{i}\right)=\left(\widehat{W}_{i}^{T}-W_{i}^{* T}\right) \Phi_{i}\left(X_{i}\right)=$

$\widetilde{W}_{i}^{T} \Phi_{i}\left(X_{i}\right)+\bar{\xi}_{i}^{*}(X)$

Due to the piecewise continuous function $f_{c i}\left(\dot{q}_{i}\right)$, we assume that $\bar{\xi}_{i}^{*}(X)$ are made up of $\xi_{i}^{*}$ and $f_{c i}\left(\dot{q}_{i}\right)$.

Combine the proposed wave-based TDPA control method and the $\mathrm{NN}$ control method, the control laws of the overall teleoperation systems are given as follows:

$\tau_{m}(t)=\hat{f}_{m}\left(X_{m}\right)+\hat{I}_{m}(t)-\left(\alpha-\alpha_{1}\right) \delta \dot{q}_{m}(t)-(1-$

$\left.b_{1} \lambda_{1}\right) \beta \delta q_{m}(t)-\left(1-b_{1} \lambda_{1}\right)\left(\gamma-\gamma_{1}\right) \delta \ddot{q}_{m}(t)-G_{m}(t)-Y_{m}(t)$

$=\hat{f}_{m}\left(X_{m}\right)-I_{A 1}-I_{B 1}-\Gamma_{1}(t)-\left(\alpha-\alpha_{1}\right) \delta \dot{q}_{m}(t)-(1-$

$\left.b_{1} \lambda_{1}\right) \beta \delta q_{m}(t)-\left(1-b_{1} \lambda_{1}\right)\left(\gamma-\gamma_{1}\right) \delta \ddot{q}_{m}(t)-G_{m}(t)-Y_{m}(t)$

$=\hat{f}_{m}\left(X_{m}\right)+\beta\left(\delta q_{s}\left(t-T_{2}(t)\right)-\delta q_{m}(t)\right)+(\alpha-$

$\left.\alpha_{1}\right)\left(\delta \dot{q}_{s}\left(t-T_{2}(t)\right)-\delta \dot{q}_{m}(t)\right)+\left(\gamma-\gamma_{1}\right)\left(\delta \ddot{q}_{s}\left(t-T_{2}(t)\right)-\right.$ $\left.\delta \ddot{q}_{m}(t)\right)-\frac{b_{2} \alpha_{1}}{\lambda_{2}}\left(\delta \dot{q}_{m}(t)-\delta \dot{q}_{m}\left(t-T_{l 2}(t)\right)\right)-$

$b_{1} \lambda_{1} \alpha \delta \dot{q}_{m}(t)-\Gamma_{1}(t)-G_{m}(t)-Y_{m}(t)$

$\tau_{s}(t)=\hat{f}_{S}\left(X_{s}\right)+\hat{V}_{s}(t)-\left(\alpha-\alpha_{1}\right) \delta \dot{q}_{s}(t)-(1-$

$\left.\frac{\lambda_{2}}{b_{2}}\right) \beta \delta q_{s}(t)-\left(1-\frac{\lambda_{2}}{b_{2}}\right)\left(\gamma-\gamma_{1}\right) \delta \ddot{q}_{s}(t)-G_{s}(t)-Y_{s}(t)$

$=\hat{f}_{S}\left(X_{S}\right)+V_{A 2}(t)-V_{B 2}(t)-\Gamma_{2}(t)-\left(\alpha-\alpha_{1}\right) \delta \dot{q}_{s}(t)-(1-$

$\left.\frac{\lambda_{2}}{b_{2}}\right) \beta \delta q_{m}(t)-\left(1-\frac{\lambda_{2}}{b_{2}}\right)\left(\gamma-\gamma_{1}\right) \delta \ddot{q}_{s}(t)-G_{s}(t)-Y_{s}(t)$

$=\hat{f}_{s}\left(X_{s}\right)+\beta\left(\delta q_{m}\left(t-T_{1}(t)\right)-\delta q_{s}(t)\right)+(\alpha-$

$\left.\alpha_{1}\right)\left(\delta \dot{q}_{m}\left(t-T_{1}(t)\right)-\delta \dot{q}_{s}(t)\right)+\left(\gamma-\gamma_{1}\right)\left(\delta \ddot{q}_{m}\left(t-T_{1}(t)\right)-\right.$

$\left.\delta \ddot{q}_{s}(t)\right)-\frac{\alpha_{1}}{b_{1} \lambda_{1}}\left(\delta \dot{q}_{s}(t)-\delta \dot{q}_{s}\left(t-T_{l 1}(t)\right)\right)-\frac{\lambda_{2} \alpha}{b_{2}} \delta \dot{q}_{s}(t)-$

$\Gamma_{2}(t)-G_{s}(t)-Y_{s}(t)$

$Y_{i}(t)$ and $G_{i}(t)$ are the designed adaptive control laws as:

$$
Y_{i}(t)=\left\{\begin{array}{c}
\frac{\delta \dot{q}_{i}(t)}{\left\|\delta \dot{q}_{i}(t)\right\|} \widehat{\Theta}_{i}(t), \text { if }\left\|\dot{q}_{i}(t)\right\| \neq 0 \\
0, \text { if }\left\|\dot{q}_{i}(t)\right\|=0
\end{array}, \dot{\widehat{\Theta}}_{\mathrm{i}}(\mathrm{t})=\left\|\delta \dot{q}_{i}(t)\right\|\right.
$$

$$
\left\{\begin{array}{c}
G_{i}(t)=k h_{i}(t) \\
\dot{h}_{i}(t)=-\frac{\gamma-\gamma_{1}}{2} \frac{\left\|\delta \ddot{q}_{i}(t)\right\|^{2}+\left\|\delta \dot{q}_{i}(t)\right\|^{2}}{\left\|h_{i}(t)\right\|^{2}} h_{i}(t)+k \delta \dot{q}_{i}(t)
\end{array}\right.
$$

The adaptive control laws $Y_{i}(t)$ are mainly used to deal with the approximation error, external positive input and unknown disturbance. $\widehat{\Theta}_{i}(\mathrm{t})$ are applied to estimate the upper bounds $\Theta_{i}$ the sum of $\mathrm{NN}$ approximate error, the bounded external disturbance $F_{i}^{*}$ and the exogenous input $\tau_{h, e}^{*}(t)$. That is, $\Theta_{i} \geq$ $\left\|\bar{\xi}_{i}^{*}(X)+F_{i}^{*} \pm \tau_{h, e}^{*}\right\|$. In the ideal situation where $\tilde{f}_{i}\left(X_{i}\right)=0$, the adaptive controllers can be considered as damping terms which may influence transparency. However, by setting $0<\delta<$ 1 , the adverse influence can be effectively reduced. The adaptive control laws $G_{i}(t)$ are applied to guarantee the stability of acceleration transmission. $h_{i}(t)$ are the states of the auxiliary system in $(58)\left(h_{i}(t) \neq 0\right) . k$ is a positive constant. setting $k<$ 1 can efficiently reduce the influence of (52) on transparency.

Based on the control laws (49)-(50), $\hat{f}_{i}\left(X_{i}\right)$ and $Y_{i}(t)$ are deployed to diminish the side effects of system uncertainties as well as the external disturbance and input. The two terms of $-b_{1} \lambda_{1} \alpha \delta \dot{q}_{m}(t)$ and $-\frac{\lambda_{2} \alpha}{b_{2}} \delta \dot{q}_{s}(t)$ are applied to guarantee the system's stability and the two relationships of $-\frac{b_{2} \alpha_{1}}{\lambda_{2}}\left(\delta \dot{q}_{m}(t)-\right.$ $\left.\delta \dot{q}_{m}\left(t-T_{l 1}(t)\right)\right)$ and $-\frac{\alpha_{1}}{b_{1} \lambda_{1}}\left(\delta \dot{q}_{s}(t)-\delta \dot{q}_{s}\left(t-T_{l 2}(t)\right)\right)$ can strengthen tracking performance and system stability. Under small time delays, these two terms are close to zero. For large time delays, they can be treated as dampers that can enhance the system stability. Also setting small value of $\alpha_{1}$ can efficiently reduce the value of the two terms. The remaining parts produce accurate position, velocity and acceleration signals tracking. The proposed control laws allow the operator to feel the position, velocity and acceleration information of the remote environment, thus, highly accurate torque tracking is expected to achieve. When large and sharply varying delays occur, $\Gamma_{1,2}(t)$ will be immediately launched to guarantee the whole system's stability. More details on setting control parameters will be introduced later. The total block diagram of the proposed teleoperation system is 
shown in Fig.3.

Theorem 1. Consider the teleoperation system (1)-(2). If the control laws are constructed by (49) and (50), the NN adaptive laws are

$$
\dot{\widehat{W}}_{i}=\Omega_{i} \Phi_{i}\left(X_{i}\right) \delta \dot{q}_{i}
$$

where $\Omega_{i}$ are the positive definite matrices. The position and velocity tracking errors will asymmetrically converge to zero in the presence of arbitrary time delays.

Proof. Consider a positive semi-definite function $V(t)$ for the system as $\mathrm{V}(\mathrm{t})=V_{1}(t)+V_{2}(t)+V_{3}(t)+V_{4}(t)+V_{5}(t)$ where

$V_{1}(t)=\frac{1}{2} \delta \dot{q}_{m}^{T}(t) M_{m}\left(q_{m}(t)\right) \delta \dot{q}_{m}(t)+$

$\frac{1}{2} \delta \dot{q}_{s}^{T}(t) M_{s}\left(q_{s}(t)\right) \delta \dot{q}_{s}(t)+\frac{1}{2} \operatorname{tr}\left(\widetilde{W}_{m}^{T} \Omega_{m}^{-1} \widetilde{W}_{m}\right)+$

$\frac{1}{2} \operatorname{tr}\left(\widetilde{W}_{s}^{T} \Omega_{s}^{-1} \widetilde{W}_{s}\right)$

$V_{2}(t)=\frac{\beta}{2}\left(\delta q_{m}(t)-\delta q_{s}(t)\right)^{T}\left(\delta q_{m}(t)-\delta q_{s}(t)\right)+\frac{1}{2}\left(\Theta_{m}-\right.$

$\left.\hat{\Theta}_{m}(t)\right)^{2}+\frac{1}{2}\left(\Theta_{s}-\hat{\Theta}_{s}(t)\right)^{2}+\frac{1}{2} h_{m}^{T}(t) h_{m}(t)+\frac{1}{2} h_{s}^{T}(t) h_{s}(t)$

$V_{3}(t)=\frac{\beta}{2} \int_{-T_{1}(t)}^{0} \int_{t+\pi}^{t} \delta^{2} \dot{q}_{m}^{T}(\eta) \dot{q}_{m}(\eta) d \eta d \pi+$

$\frac{\beta}{2} \int_{-T_{2}(t)}^{0} \int_{t+\pi}^{t} \delta^{2} \dot{q}_{s}^{T}(\eta) \dot{q}_{s}(\eta) d \eta d \pi$

$V_{4}(t)=\frac{\alpha-\alpha_{1}}{2} \int_{t-T_{1}(t)}^{t} \delta^{2} \dot{q}_{m}^{T}(\eta) \dot{q}_{m}(\eta) d \eta+$

$\frac{\alpha-\alpha_{1}}{2} \int_{t-T_{2}(t)}^{t} \delta^{2} \dot{q}_{s}^{T}(\eta) \dot{q}_{s}(\eta) d \eta+$

$\frac{\gamma-\gamma_{1}}{2} \int_{t-T_{1}(t)}^{t} \delta^{2} \ddot{q}_{m}^{T}(\eta) \ddot{q}_{m}(\eta) d \eta+$

$\frac{\gamma-\gamma_{1}}{2} \int_{t-T_{2}(t)}^{t} \delta^{2} \ddot{q}_{s}^{T}(\eta) \ddot{q}_{s}(\eta) d \eta+$

$\frac{b_{2} \alpha_{1}}{2 \lambda_{2}} \int_{t-T_{l 2}(t)}^{t} \delta^{2} \dot{q}_{m}^{T}(\eta) \dot{q}_{m}(\eta) d \eta+$

$\frac{\alpha_{1}}{2 b_{1} \lambda_{1}} \int_{t-T_{l 1}(t)}^{t} \delta^{2} \dot{q}_{s}^{T}(\eta) \dot{q}_{s}(\eta) d \eta$

$V_{5}(t)=\delta^{2} \dot{q}_{m}^{T}(t) \frac{M_{h} / \delta+\gamma-\gamma_{1}}{2} \dot{q}_{m}(t)+\delta^{2} \dot{q}_{s}^{T}(t) \frac{M_{e} / \delta+\gamma-\gamma_{1}}{2} \dot{q}_{s}(t)$

Using property 2 in Section 2, the control laws (49) and (50), the modelled human and environmental torques (5) and (6), and the NNs adaptive laws (53), the time derivative of $V_{1}(t)$ can be written as:

$\dot{V}_{1}(t)=\delta \dot{q}_{m}^{T}(t)\left(\hat{f}_{m}\left(X_{m}\right)-f_{m}\left(X_{m}\right)-B_{h} \dot{q}_{m}(t)-M_{h} \ddot{q}_{m}(t)+\right.$ $\tau_{h}^{*}(t)+F_{m}^{*}+\beta\left(\delta q_{s}\left(t-T_{2}(t)\right)-\delta q_{m}(t)\right)+(\alpha-$ $\left.\alpha_{1}\right)\left(\delta \dot{q}_{s}\left(t-T_{2}(t)\right)-\delta \dot{q}_{m}(t)\right)+\left(\gamma-\gamma_{1}\right)\left(\delta \ddot{q}_{s}\left(t-T_{2}(t)\right)-\right.$ $\left.\delta \ddot{q}_{m}(t)\right)-2 b_{1} \lambda_{1} \alpha \delta \dot{q}_{m}(t)-\frac{b_{2} \alpha_{1}}{\lambda_{2}}\left(\delta \dot{q}_{m}(t)-\delta \dot{q}_{m}(t-\right.$ $\left.\left.\left.T_{l 2}(t)\right)\right)-\Gamma_{1}(t)-\frac{\delta \dot{q}_{m}(t)}{\left\|\delta \dot{q}_{m}(t)\right\|} \widehat{\Theta}_{m}(t)-G_{m}(t)\right)+$ $\delta \dot{q}_{s}^{T}(t)\left(\hat{f}_{s}\left(X_{s}\right)-f_{s}\left(X_{s}\right)-B_{e} \dot{q}_{s}(t)-M_{e} \ddot{q}_{s}(t)+F_{s}^{*}-\tau_{e}^{*}(t)+\right.$ $\beta\left(\delta q_{m}\left(t-T_{1}(t)\right)-\delta q_{s}(t)\right)+\left(\alpha-\alpha_{1}\right)\left(\delta \dot{q}_{m}\left(t-T_{1}(t)\right)-\right.$ $\left.\delta \dot{q}_{s}(t)\right)+\left(\gamma-\gamma_{1}\right)\left(\delta \ddot{q}_{m}\left(t-T_{1}(t)\right)-\delta \ddot{q}_{s}(t)\right)-$ $\frac{2 \lambda_{2} \alpha}{b_{2}} \delta \dot{q}_{s}(t)-\frac{\alpha_{1}}{b_{1} \lambda_{1}}\left(\delta \dot{q}_{s}(t)-\delta \dot{q}_{s}\left(t-T_{l 1}(t)\right)\right)-\Gamma_{2}(t)-$ $\left.\frac{\delta \dot{q}_{s}(t)}{\left\|\delta \dot{q}_{s}(t)\right\|} \hat{\Theta}_{S}(t)-G_{s}(t)\right)-\operatorname{tr}\left(\widetilde{W}_{m}^{T} \Phi_{m}\left(X_{m}\right) \delta \dot{q}_{m}(t)\right)-$ $\operatorname{tr}\left(\widetilde{W}_{s}^{T} \Phi_{s}\left(X_{s}\right) \delta \dot{q}_{s}(t)\right)$
Also, the time derivative of $V_{2}(t)$ is given by

$\dot{V}_{2}(t)=\beta \delta \dot{q}_{m}^{T}(t)\left(\delta q_{m}(t)-\delta q_{s}\left(t-T_{2}(t)\right)\right)+$

$\beta \delta \dot{q}_{s}^{T}(t)\left(\delta q_{s}(t)-\delta q_{m}\left(t-T_{1}(t)\right)\right)-$

$\beta \delta \dot{q}_{m}^{T}(t) \int_{t-T_{2}(t)}^{t} \delta \dot{q}_{s}(\eta) d \eta-\beta \delta \dot{q}_{s}^{T}(t) \int_{t-T_{1}(t)}^{t} \dot{q}_{m}(\eta) d \eta+$

$\left(\hat{\Theta}_{m}(t)-\Theta_{m}\right) \dot{\hat{\Theta}}_{m}(t)+\left(\hat{\Theta}_{s}(t)-\Theta_{s}\right) \dot{\hat{\Theta}}_{s}(t)+h_{m}(t) \dot{h}_{m}(t)+$ $+h_{s}(t) \dot{h}_{s}(t)$

After algebraic manipulations, time derivative of $V_{3}(t)$ is found to satisfy

$\dot{V}_{3}(t) \leq T_{1}^{\max } \dot{q}_{m}^{T}(t) \frac{\beta \delta^{2}}{2} \dot{q}_{m}(t)-\frac{\beta}{2} \int_{t-T_{1}(t)}^{t} \delta^{2} \dot{q}_{m}^{T}(\eta) \dot{q}_{m}(\eta) d \eta+$ $T_{2}^{\max } \dot{q}_{s}^{T}(t) \frac{\beta \delta^{2}}{2} \dot{q}_{s}(t)-\frac{\beta}{2} \int_{t-T_{2}(t)}^{t} \delta^{2} \dot{q}_{s}^{T}(\eta) \dot{q}_{s}(\eta) d \eta$

The time derivative of $V_{4}(t)$ can also be written as

$\dot{V}_{4}(t)=\frac{\alpha-\alpha_{1}}{2}\left(\delta^{2} \dot{q}_{m}^{T}(t) \dot{q}_{m}(t)-\delta^{2} \dot{q}_{m}^{T}\left(t-T_{1}(t)\right) \dot{q}_{m}(t-\right.$

$\left.\left.T_{1}(t)\right)+\dot{T}_{1}(t) \delta^{2} \dot{q}_{m}^{T}\left(t-T_{1}(t)\right) \dot{q}_{m}\left(t-T_{1}(t)\right)\right)+$ $\frac{\alpha-\alpha_{1}}{2}\left(\delta^{2} \dot{q}_{s}^{T}(t) \dot{q}_{s}(t)-\delta^{2} \dot{q}_{s}^{T}\left(t-T_{2}(t)\right) \dot{q}_{s}\left(t-T_{2}(t)\right)+\right.$ $\left.\dot{T}_{2}(t) \delta^{2} \dot{q}_{s}^{T}\left(t-T_{2}(t)\right) \dot{q}_{s}\left(t-T_{2}(t)\right)\right)+\frac{\gamma-\gamma_{1}}{2}\left(\delta^{2} \ddot{q}_{m}^{T}(t) \ddot{q}_{m}(t)-\right.$ $\delta^{2} \ddot{q}_{m}^{T}\left(t-T_{1}(t)\right) \ddot{q}_{m}\left(t-T_{1}(t)\right)+\dot{T}_{1}(t) \delta^{2} \ddot{q}_{m}^{T}(t-$

$\left.\left.T_{1}(t)\right) \ddot{q}_{m}\left(t-T_{1}(t)\right)\right)+\frac{\gamma-\gamma_{1}}{2}\left(\delta^{2} \ddot{q}_{s}^{T}(t) \ddot{q}_{s}(t)-\delta^{2} \ddot{q}_{s}^{T}(t-\right.$

$\left.\left.T_{2}(t)\right) \ddot{q}_{s}\left(t-T_{2}(t)\right)+\dot{T}_{2}(t) \delta^{2} \ddot{q}_{s}^{T}\left(t-T_{2}(t)\right) \ddot{q}_{s}\left(t-T_{2}(t)\right)\right)+$ $\frac{b_{2} \alpha_{1}}{2 \lambda_{2}}\left(\delta^{2} \dot{q}_{m}^{T}(t) \dot{q}_{m}(t)-\delta^{2} \dot{q}_{m}^{T}\left(t-T_{l 2}(t)\right) \dot{q}_{m}\left(t-T_{l 2}(t)\right)+\right.$

$\left.\dot{T}_{l 2}(t) \delta^{2} \dot{q}_{m}^{T}\left(t-T_{l 2}(t)\right) \dot{q}_{m}\left(t-T_{l 2}(t)\right)\right)+$

$\frac{\alpha_{1}}{2 b_{1} \lambda_{1}}\left(\delta^{2} \dot{q}_{s}^{T}(t) \dot{q}_{s}(t)-\delta^{2} \dot{q}_{s}^{T}\left(t-T_{l 1}(t)\right) \dot{q}_{s}\left(t-T_{l 1}(t)\right)+\right.$

$\left.\dot{T}_{l 1}(t) \delta^{2} \dot{q}_{s}^{T}\left(t-T_{l 1}(t)\right) \dot{q}_{s}\left(t-T_{l 1}(t)\right)\right)$

The differential of $V_{5}(t)$ is

$\dot{V}_{5}(t)=\dot{q}_{m}^{T}(t) \delta M_{h} \ddot{q}_{m}(t)+\dot{q}_{s}^{T}(t) \delta M_{e} \ddot{q}_{s}(t)+\dot{q}_{m}^{T}(t) \delta^{2}(\gamma-$

$\left.\gamma_{1}\right) \ddot{q}_{m}(t)+\dot{q}_{s}^{T}(t) \delta^{2}\left(\gamma-\gamma_{1}\right) \ddot{q}_{s}(t)$

By setting $\sigma_{1-2}$ large enough to make sure

$$
\begin{aligned}
& \dot{q}_{m}^{T}(t) \dot{q}_{m}(t)+\sigma_{1}>\dot{q}_{m}^{T}\left(t-T_{l 2}(t)\right) \dot{q}_{m}\left(t-T_{l 2}(t)\right) \\
& \dot{q}_{s}^{T}(t) \dot{q}_{s}(t)+\sigma_{2}>\dot{q}_{s}^{T}\left(t-T_{l 1}(t)\right) \dot{q}_{s}\left(t-T_{l 1}(t)\right)
\end{aligned}
$$

using the following inequalities from Lemma 1 in [37]

$-2 \dot{q}_{m}^{T}(t) \int_{t-T_{2}(t)}^{t} \dot{q}_{s}(\eta) d \eta-\int_{t-T_{2}(t)}^{t} \dot{q}_{s}^{T}(\eta) \dot{q}_{s}(\eta) d \eta \leq$ $T_{2}^{\max } \dot{q}_{m}^{T}(t) \dot{q}_{m}(t)$

$-2 \dot{q}_{s}^{T}(t) \int_{t-T_{1}(t)}^{t} \dot{q}_{m}(\eta) d \eta-\int_{t-T_{1}(t)}^{t} \dot{q}_{m}^{T}(\eta) \dot{q}_{m}(\eta) d \eta \leq$ $T_{1}^{\max } \dot{q}_{s}^{T}(t) \dot{q}_{s}(t)$

and with the adaptive laws (51)-(52), it can be derived that

$\dot{V}(t)<-\delta \dot{q}_{m}^{T}(t)\left(b_{1} \lambda_{1} \alpha-\frac{\beta}{2}\left(\bar{T}_{1}+\bar{T}_{2}+\bar{\varepsilon}_{1}+\bar{\varepsilon}_{2}\right)\right) \delta \dot{q}_{m}(t)-$

$\delta \dot{q}_{s}^{T}(t)\left(\frac{\lambda_{2} \alpha}{b_{2}}-\frac{\beta}{2}\left(\bar{T}_{1}+\bar{T}_{2}+\bar{\varepsilon}_{1}+\bar{\varepsilon}_{2}\right)\right) \delta \dot{q}_{s}(t)-$

$\frac{\alpha-\alpha_{1}}{2}\left(\delta \dot{q}_{m}(t)-\delta \dot{q}_{s}\left(t-T_{2}(t)\right)\right)^{T}\left(\delta \dot{q}_{m}(t)-\delta \dot{q}_{s}(t-\right.$

$\left.\left.T_{2}(t)\right)\right)-\frac{\alpha-\alpha_{1}}{2}\left(\delta \dot{q}_{s}(t)-\delta \dot{q}_{m}\left(t-T_{1}(t)\right)\right)^{T}\left(\delta \dot{q}_{s}(t)-\right.$

$\left.\delta \dot{q}_{m}\left(t-T_{2}(t)\right)\right)-\frac{\gamma-\gamma_{1}}{2}\left(\delta \dot{q}_{m}(t)-\delta \ddot{q}_{s}(t-\right.$ 
$\left.\left.T_{2}(t)\right)\right)^{T}\left(\delta \dot{q}_{m}(t)-\delta \ddot{q}_{s}\left(t-T_{2}(t)\right)\right)-\frac{\gamma-\gamma_{1}}{2}\left(\delta \dot{q}_{s}(t)-\right.$

$\left.\delta \ddot{q}_{m}\left(t-T_{1}(t)\right)\right)^{T}\left(\delta \dot{q}_{s}(t)-\delta \ddot{q}_{m}\left(t-T_{2}(t)\right)\right)-$

$\frac{b_{2} \alpha_{1}}{2 \lambda_{2}}\left(\delta \dot{q}_{m}(t)-\delta \dot{q}_{m}\left(t-T_{l 2}(t)\right)\right)^{T}\left(\delta \dot{q}_{m}(t)-\delta \dot{q}_{m}(t-\right.$

$\left.\left.T_{l 2}(t)\right)\right)-\frac{\alpha_{1}}{2 b_{1} \lambda_{1}}\left(\delta \dot{q}_{s}(t)-\delta \dot{q}_{s}\left(t-T_{l 1}(t)\right)\right)^{T}\left(\delta \dot{q}_{s}(t)-\right.$

$\left.\delta \dot{q}_{s}\left(t-T_{l 1}(t)\right)\right)+\frac{\left(\alpha-\alpha_{1}\right) \dot{T}_{1}(t) \delta^{2}}{2} \dot{q}_{m}^{T}\left(t-T_{1}(t)\right) \dot{q}_{m}(t-$

$\left.T_{1}(t)\right)+\frac{\left(\alpha-\alpha_{1}\right) \dot{T}_{2}(t) \delta^{2}}{2} \dot{q}_{s}^{T}\left(t-T_{2}(t)\right) \dot{q}_{s}\left(t-T_{2}(t)\right)+$

$\frac{\left(\gamma-\gamma_{1}\right) \dot{T}_{1}(t) \delta^{2}}{2} \ddot{q}_{m}^{T}\left(t-T_{1}(t)\right) \ddot{q}_{m}\left(t-T_{1}(t)\right)+\frac{\left(\gamma-\gamma_{1}\right) \dot{T}_{2}(t) \delta^{2}}{2} \ddot{q}_{s}^{T}(t-$

$\left.T_{2}(t)\right) \ddot{q}_{s}\left(t-T_{2}(t)\right)+\frac{b_{2} \alpha_{1} \dot{T}_{l 2}(t) \delta^{2}}{2 \lambda_{2}}\left(\dot{q}_{m}^{T}(t) \dot{q}_{m}(t)+\sigma_{1}\right)+$

$\frac{\alpha_{1} \dot{T}_{l 1}(t) \delta^{2}}{2 b_{1} \lambda_{1}}\left(\dot{q}_{s}^{T}(t) \dot{q}_{s}(t)+\sigma_{2}\right)-\delta \dot{q}_{m}^{T}(t) \Gamma_{1}(t)-\delta \dot{q}_{s}^{T}(t) \Gamma_{2}(t)-$

$\delta \dot{q}_{m}^{T}(t) B_{h} \dot{q}_{m}(t)-\delta \dot{q}_{s}^{T}(t) B_{e} \dot{q}_{s}(t)+\delta \dot{q}_{m}^{T}(t)\left(\bar{\xi}_{m}^{*}(X)+\right.$

$\left.\tau_{h}^{*}(t)+F_{m}^{*}-\frac{\dot{q}_{m}(t)}{\left\|\dot{q}_{m}(t)\right\|} \hat{\Theta}_{m}(t)\right)+\delta \dot{q}_{s}^{T}(t)\left(\bar{\xi}_{s}^{*}(X)-\tau_{e}^{*}(t)+F_{s}^{*}-\right.$

$\left.\frac{\delta \dot{q}_{s}(t)}{\left\|\delta \dot{q}_{s}(t)\right\|} \hat{\Theta}_{s}(t)\right)+\left(\widehat{\Theta}_{m}(t)-\Theta_{m}\right)\left\|\delta \dot{q}_{m}(t)\right\|+\left(\widehat{\Theta}_{s}(t)-\right.$

$\left.\Theta_{s}\right)\left\|\delta \dot{q}_{m}(t)\right\|$

Substituting the upper bounds $\Theta_{i}$ of $\left\|\bar{\xi}_{i}^{*}(X)+F_{i}^{*} \pm \tau_{h, e}^{*}\right\|$ into (68), the final four terms in (68) can be removed. The Lyapunov approach requires $\dot{V}(t)$ to be negative semi-definite. In the presence of constant time delays, $\dot{T}_{1,2}(t)$ and $\bar{\varepsilon}_{1,2}$ are zero. Also, the passivity controllers do not take effect so that $\Gamma_{1}(t)$ and $\quad \Gamma_{2}(t) \quad$ are $\quad$ zero. $\quad-\frac{b_{2} \alpha_{1}}{2 \lambda_{2}}\left(\delta \dot{q}_{m}(t)-\delta \dot{q}_{m}(t-\right.$ $\left.\left.T_{l 2}(t)\right)\right)^{T}\left(\delta \dot{q}_{m}(t)-\delta \dot{q}_{m}\left(t-T_{l 2}(t)\right)\right)$ and $-\frac{\alpha_{1}}{2 b_{1} \lambda_{1}}\left(\delta \dot{q}_{s}(t)-\right.$ $\left.\delta \dot{q}_{s}\left(t-T_{l 1}(t)\right)\right)^{T}\left(\delta \dot{q}_{s}(t)-\delta \dot{q}_{s}\left(t-T_{l 1}(t)\right)\right)$ can be seen as $-\frac{b_{2} \alpha_{1}}{2 \lambda_{2}} \zeta_{a} \delta^{2} \dot{q}_{m}^{T}(t) \dot{q}_{m}(t)$ and $-\frac{\alpha_{1}}{2 b_{1} \lambda_{1}} \zeta_{b} \delta^{2} \dot{q}_{s}^{T}(t) \dot{q}_{s}(t) \quad$ with $\zeta_{a, b} \geq 0$. Under small time delays, $\zeta_{a, b} \rightarrow 0 . \dot{V}(t)$ can be guaranteed to be negative semi-definite by properly tuning $b_{1,2}$, $\lambda_{1,2}, \alpha, \alpha_{1}$ and $\beta$ to make sure

$$
\begin{aligned}
& b_{1} \lambda_{1} \alpha+\frac{b_{2} \alpha_{1}}{2 \lambda_{2}} \zeta_{a} \geq \frac{\beta}{2}\left(\bar{T}_{1}+\bar{T}_{2}\right) \\
& \frac{\lambda_{2} \alpha}{b_{2}}+\frac{\alpha_{1}}{2 b_{1} \lambda_{1}} \zeta_{b} \geq \frac{\beta}{2}\left(\bar{T}_{1}+\bar{T}_{2}\right)
\end{aligned}
$$

When the time delay is varying, the passivity controllers are launched by the passivity observers, substituting (42)-(43) in to (68), the biased terms $\frac{\left(\alpha-\alpha_{1}\right) \dot{T}_{1}(t) \delta^{2}}{2} \dot{q}_{m}^{T}\left(t-T_{1}(t)\right) \dot{q}_{m}(t-$ $\left.T_{1}(t)\right)+\frac{\left(\alpha-\alpha_{1}\right) \dot{T}_{2}(t) \delta^{2}}{2} \dot{q}_{s}^{T}\left(t-T_{2}(t)\right) \dot{q}_{s}\left(t-T_{2}(t)\right)+$ $\frac{\left(\gamma-\gamma_{1}\right) \dot{T}_{1}(t) \delta^{2}}{2} \ddot{q}_{m}^{T}\left(t-T_{1}(t)\right) \ddot{q}_{m}\left(t-T_{1}(t)\right)+\frac{\left(\gamma-\gamma_{1}\right) \dot{T}_{2}(t) \delta^{2}}{2} \ddot{q}_{s}^{T}(t-$ $\left.T_{2}(t)\right) \ddot{q}_{s}\left(t-T_{2}(t)\right)+\frac{b_{2} \alpha_{1} \dot{T}_{l 2}(t) \delta^{2}}{2 \lambda_{2}}\left(\dot{q}_{m}^{T}(t) \dot{q}_{m}(t)+\sigma_{1}\right)+$ $\frac{\alpha_{1} \dot{T}_{l 1}(t) \delta^{2}}{2 b_{1} \lambda_{1}}\left(\dot{q}_{s}^{T}(t) \dot{q}_{s}(t)+\sigma_{2}\right)$ caused by the time varying delays in $(68)$ are directly compensated by $-\delta \dot{q}_{m}^{T}(t) \Gamma_{1}(t)-$ $\delta \dot{q}_{s}^{T}(t) \Gamma_{2}(t)$. No extra parameters need to be tuned when the time delays vary and $\dot{\mathrm{V}}(t)$ is still negative semi-definite.

Integrating both sides of (68), we get:

$+\infty>V(0) \geq V(0)-V(t)>\int_{0}^{t}\left(\frac{\alpha-\alpha_{1}}{2}\left(\delta \dot{q}_{m}(t)-\delta \dot{q}_{s}(t-\right.\right.$

$$
\begin{aligned}
& \left.\left.T_{2}(t)\right)\right)^{T}\left(\delta \dot{q}_{m}(t)-\delta \dot{q}_{s}\left(t-T_{2}(t)\right)\right)+\frac{\alpha-\alpha_{1}}{2}\left(\delta \dot{q}_{s}(t)-\right. \\
& \left.\delta \dot{q}_{m}\left(t-T_{1}(t)\right)\right)^{T}\left(\delta \dot{q}_{s}(t)-\delta \dot{q}_{m}\left(t-T_{2}(t)\right)\right)+ \\
& \frac{\gamma-\gamma_{1}}{2}\left(\delta \dot{q}_{m}(t)-\delta \ddot{q}_{s}\left(t-T_{2}(t)\right)\right)^{T}\left(\delta \dot{q}_{m}(t)-\delta \ddot{q}_{s}(t-\right. \\
& \left.\left.T_{2}(t)\right)\right)+\frac{\gamma-\gamma_{1}}{2}\left(\delta \dot{q}_{s}(t)-\delta \ddot{q}_{m}\left(t-T_{1}(t)\right)\right)^{T}\left(\delta \dot{q}_{s}(t)-\right. \\
& \left.\delta \ddot{q}_{m}\left(t-T_{2}(t)\right)\right)+\frac{b_{2} \alpha_{1}}{2 \lambda_{2}}\left(\delta \dot{q}_{m}(t)-\delta \dot{q}_{m}(t-\right. \\
& \left.\left.T_{l 2}(t)\right)\right)^{T}\left(\delta \dot{q}_{m}(t)-\delta \dot{q}_{m}\left(t-T_{l 2}(t)\right)\right)+\frac{\alpha_{1}}{2 b_{1} \lambda_{1}}\left(\delta \dot{q}_{s}(t)-\right. \\
& \left.\delta \dot{q}_{s}\left(t-T_{l 1}(t)\right)\right)^{T}\left(\delta \dot{q}_{s}(t)-\delta \dot{q}_{s}\left(t-T_{l 1}(t)\right)\right)+ \\
& \left.\delta \dot{q}_{m}^{T}(t) B_{h} \dot{q}_{m}(t)+\delta \dot{q}_{s}^{T}(t) B_{e} \dot{q}_{s}(t)\right) d t
\end{aligned}
$$

Therefore, from $V(t) \geq 0$ and $\dot{V}(t) \leq 0$, it is true that $\widetilde{W}_{m}$ and $\widetilde{W}_{s} \in L_{\infty}, \quad \dot{q}_{m}(t)$ and $\dot{q}_{s}(t) \in L_{2} .\left(\dot{q}_{m}(t)-\dot{q}_{s}(t-\right.$ $\left.\left.T_{2}(t)\right)\right), \quad\left(\dot{q}_{s}(t)-\dot{q}_{m}\left(t-T_{2}(t)\right)\right), \quad\left(\delta \dot{q}_{m}(t)-\delta \ddot{q}_{s}(t-\right.$ $\left.\left.T_{2}(t)\right)\right),\left(\delta \dot{q}_{s}(t)-\delta \ddot{q}_{m}\left(t-T_{2}(t)\right)\right), \quad\left(\dot{q}_{m}(t)-\dot{q}_{m}(t-\right.$ $\left.\left.T_{l 2}(t)\right)\right),\left(\dot{q}_{s}(t)-\dot{q}_{s}\left(t-T_{l 1}(t)\right)\right) \in L_{2}$. Using the fact that $q_{m}(t)-q_{s}\left(t-T_{2}(t)\right)=q_{m}(t)-q_{s}(t)+\int_{t-T_{2}(t)}^{t} \dot{q}_{s}(t) d t$ $q_{s}(t)-q_{m}\left(t-T_{1}(t)\right)=q_{s}(t)-q_{m}(t)+\int_{t-T_{1}(t)}^{t} \dot{q}_{1}(t) d t$ and using Cauchy-Schwarz inequality $\int_{t-T_{2}(t)}^{t} \dot{q}_{s}(t) d t \leq$ $\sqrt{T_{2}(t)} \dot{q}_{s}(t)$ and $\int_{t-T_{1}(t)}^{t} \dot{q}_{m}(t) d t \leq \sqrt{T_{1}(t)} \dot{q}_{m}(t)$, we can get $q_{m}(t)-q_{s}\left(t-T_{2}(t)\right), q_{s}(t)-q_{m}\left(t-T_{1}(t)\right) \in L_{\infty}$.

The system's dynamic model can also be written as:

$\delta \ddot{q}_{i}=M_{i}^{-1}\left(q_{i}\right)\left[\tau_{i} \pm \tau_{h, e}^{*}(t)-B_{h, e} \dot{q}_{i}(t)-M_{h, e} \ddot{q}_{i}(t)+F_{i}^{*}-\right.$ $\left.f_{i}\left(X_{i}\right)-C_{i}\left(q_{i}, \dot{q}_{i}\right) \delta \dot{q}_{i}\right]$

Differentiating both sides of (72):

$$
\begin{aligned}
& \frac{d}{d t} \delta \ddot{q}_{i}=\frac{d}{d t}\left(M_{i}^{-1}\left(q_{i}\right)\right)\left[\tau_{i} \pm \tau_{h, e}^{*}(t)-B_{h, e} \dot{q}_{i}(t)-M_{h, e} \ddot{q}_{i}(t)+\right. \\
& \left.F_{i}^{*}-f_{i}\left(X_{i}\right)-C_{i}\left(q_{i}, \dot{q}_{i}\right) \delta \dot{q}_{i}\right]+M_{i}^{-1}\left(q_{i}\right) \frac{d}{d t}\left[\tau_{i} \pm \tau_{h, e}^{*}(t)-\right. \\
& \left.B_{h, e} \dot{q}_{i}(t)-M_{h, e} \ddot{q}_{i}(t)+F_{i}^{*}-f_{i}\left(X_{i}\right)-C_{i}\left(q_{i}, \dot{q}_{i}\right) \delta \dot{q}_{i}\right]
\end{aligned}
$$

For the first term of the right side of (73), we have

$$
\frac{d}{d t}\left(M_{i}^{-1}\right)=-M_{i}^{-1} \dot{M}_{i} M_{i}^{-1}=-M_{i}^{-1}\left(C_{i}+C_{i}^{T}\right) M_{i}^{-1}
$$

According to Properties 1 and $3, \frac{d}{d t}\left(M_{i}^{-1}\right)$ is bounded. Based on Property 4, the terms in bracket of (74) are also bounded. Therefore, $\frac{d}{d t} \ddot{q}_{i}(t) \in L_{\infty}$ and $\ddot{\mathrm{q}}_{\mathrm{i}}(\mathrm{t})$ are uniformly continuous $\left(\int_{0}^{t} \ddot{q}_{i}(\eta) d \eta=\dot{q}_{i}(t)-\dot{q}_{i}(0)\right)$. Since $\dot{q}_{i}(t) \rightarrow 0$, it can be concluded that $\ddot{q}_{i}(t) \rightarrow 0$ based on Barbǎlat's Lemma.

\section{Experimental work}

The teleoperation system used to validate the proposed algorithm consists of two 3-DOF Phantom manipulators: Phantom Omni and Phantom Desktop (Sensible Technologies, Inc., Wilmington, MA) as shown in Fig.3. The two haptic devise are connected by two computers that are directly connected via a network cable and network cards. The Matlab software is applied to establish the proposed control system. To further enlarge and tune the value of the time delays, Simulink time delay blocks are also applied. During the experimental process, the control loop is configured 
as a $1 \mathrm{kHz}$ sampling rate. The general control parameters are configured as: $b_{1}=b_{2}=2, \alpha=[15,15,15]^{T}, \quad \beta=$ $[15,15,15]^{T}, \alpha_{1}=[2.5,2.5,2.5]^{T}, \gamma=[60,60,60]^{T}, \gamma_{1}=$ $[10,10,10]^{T} \delta=0.2, \Omega_{s}=\Omega_{m}=[25,25,25]^{T} . \sigma_{1}=\sigma_{2}=$ 0.25. $\varrho=4, k=0.1$. We set the number of the neuron $\kappa=7$. The center of the RBF is set as $C=0.5 \times$ ones $(9,7)$ and the width of the RBF is set as $H=0.1 \times$ ones $(7,1)$. The parameters relating to the time delays will be introduced in each experiment.
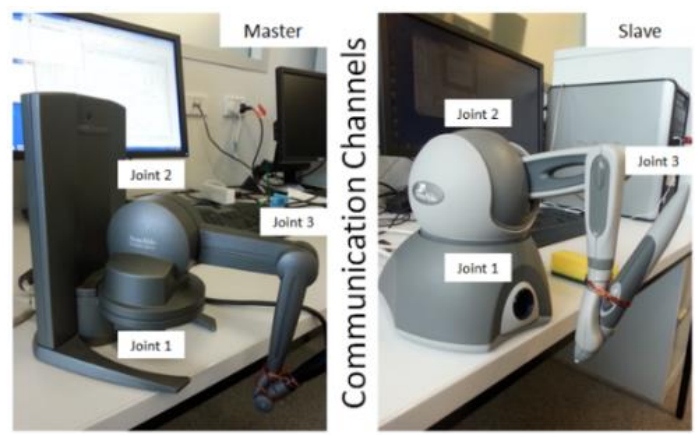

Fig.4. Experimental setup

\subsection{Innovative passivity observers}

The experiments conducted clearly demonstrate the novelty and contribution of the proposed passivity observers. The proposed system is compared with a TDPA-based system in [35]. The time delays are constant and $T_{1}$ is $200 \mathrm{~ms}, T_{2}$ is $100 \mathrm{~ms}$. According to (69) and (70), we set $\lambda_{1}=0.075, \lambda_{2}=0.3, \bar{\mu}_{1}=\bar{\mu}_{2}=\bar{\varepsilon}_{1}=$ $\bar{\varepsilon}_{2}=0$. In the system in [35], the slave PD controllers are chosen as $K_{p}=10$ and $K_{d}=5$, and $b$ in the passivity observers is set to 2.5 .

Fig. 5 shows the position tracking, torque tracking and observed power of the two systems during free motion. Even under small constant time delays whilst the rate of the time delays are zero, based on (16), the power observed in the master side is still nonpositive so that the passivity controllers are still launched to reduce tracking performance. The launched passivity controllers regrade the position tracking and make the system over-damped so that the human operator can feel large feedback forces during free motion. As shown in Fig.5, the slave cannot quickly and closely track the master in the presence of such small delays in the system in [35]. Unlike [35], the power signals observed on the master and the slave sides of the proposed system are positive owning to the designed wave-based passivity observer, and the passivity controllers are not launched. Therefore, the slave can closely track the master and the human operator can hardly feel the feedback force.

On the other hand, Fig.6 displays the position tracking, torque tracking, and observed power signals of the two systems during hard contact. Based on (17), the power observed on the slave side in the system in [35] is definitely non-positive. Therefore, large torque tracking errors are caused by the passivity controllers. By contrast, the power signals observed in the proposed system are non-negative resulting in accurate torque tracking during hard contact. From these two diagrams, it can be observed that the proposed wave-based passivity observers makes the proposed system less conservative compared the conventional powerbased systems and guarantees the system high tracking performance in the presence of constant time delays.
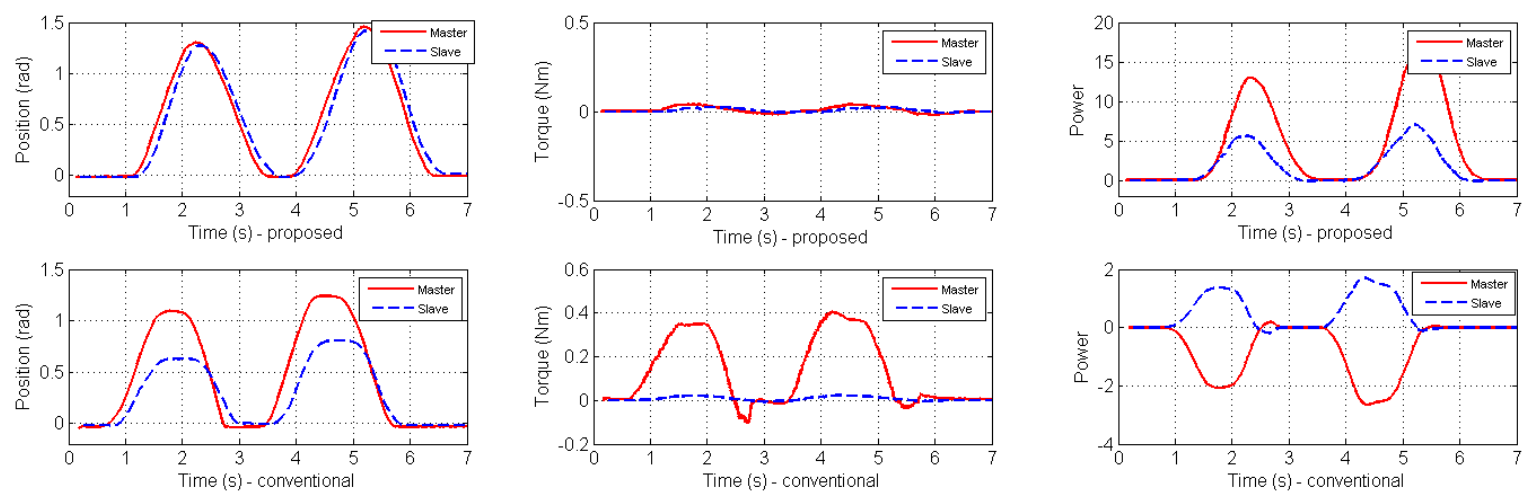

Fig.5. Free motion under constant time delays (Comparison between [35] and our system)
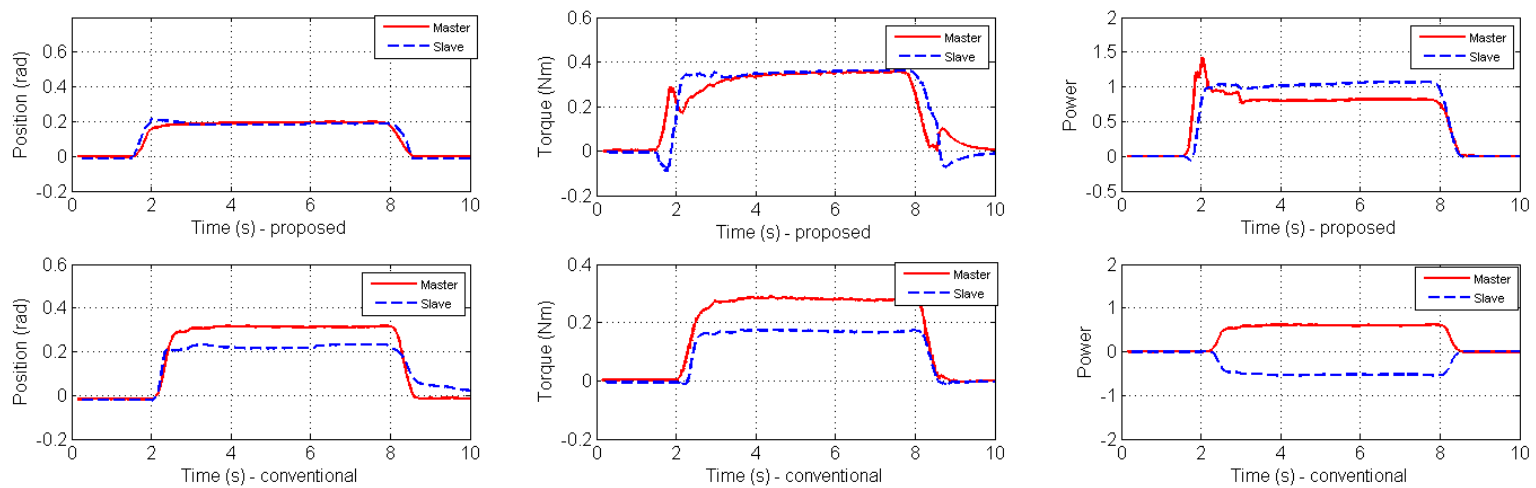

Fig.6. Hard contact under constant time delays (Comparison between [35] and our system) 

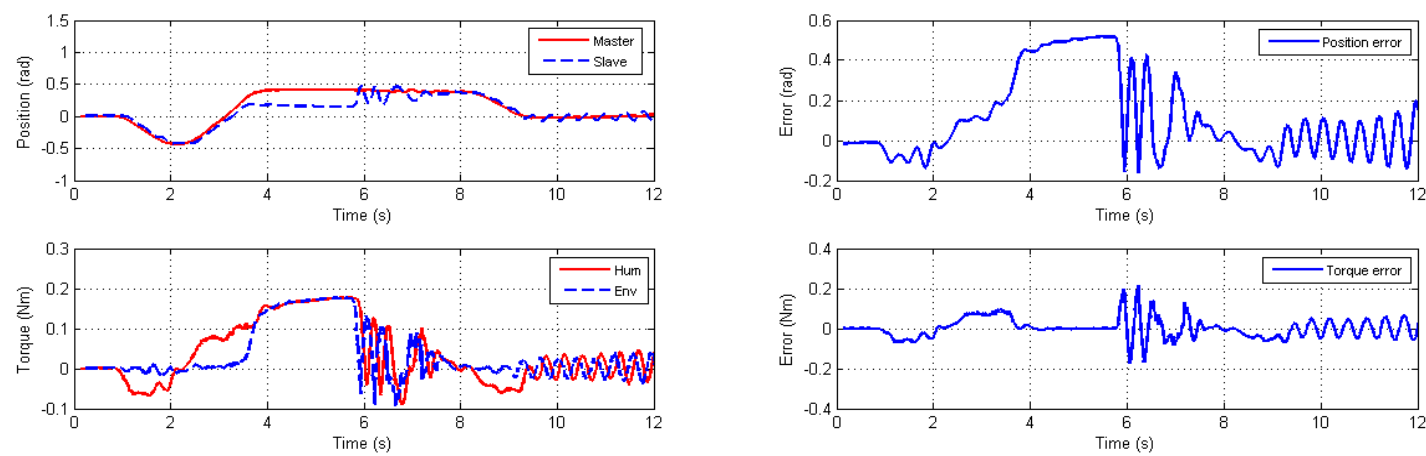

Fig.7. Contact to a reverse wall under slowly varying delays (wave-based system in [11])
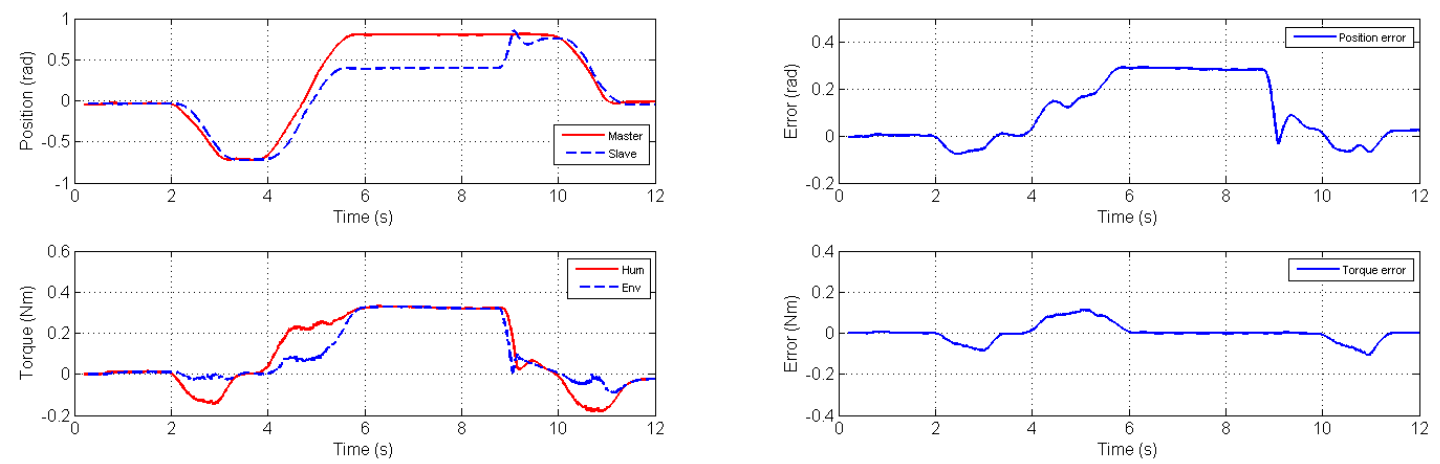

Fig.8. Contact to a reverse wall under slowly varying delays (our system)

\subsection{Eliminating wave reflection}

In this experiment, the proposed system is compared against the wave-based system proposed in [11] in order to show its effectiveness in eliminating wave reflection Figs.7 and 8 show the position and torque tracking and their relating tracking errors when the slave is in contact with a reverse wall. The time delay in this experiment is around $900 \mathrm{~ms}$ with 100 variations, and its rate is around 0.2. According to (69) and (70), we set $\lambda_{1}=0.45$, $\lambda_{2}=1.8, \quad \bar{\mu}_{1}=\bar{\mu}_{2}=0.2, \bar{\varepsilon}_{1}=\bar{\varepsilon}_{2}=0.2$. The wave-based system in [11] uses a traditional wave transformation with impedance matching to encode the velocity and position signals. Based on recommended values, we set $K_{m}=K_{s}=b=4$. The extra energy caused by time-varying delays in their system is eliminated by applying the scaling gain $\sqrt{1-\bar{\mu}_{1,2}}$.

At first, the slave robots in both systems are in free motion and both have accurate position tracking. Then the slave robots come in contacts with the reverse wall, in which both systems achieve accurate torque tracking. Therefore, it can be stated that both systems have good steady state performance. After about 2 seconds, the wall is suddenly removed, causing a sudden change in the environment. As a result, the impedances-matching approach of the system in [11] fails to work at the transient state to the extent that the wave-reflections are restored causing large perturbations that adversely affect the position and torque tracking performances of the system in [11] as shown in Fig.7. By contrast, based on the designed wave variables in (20)-(23), the outgoing signals do not contain necessary signals and the wave reflections are eliminated. Therefore, the proposed system has better performance on the transient state and the position error directly returned to zero after removing the wall as shown in Fig.8.

\subsection{Performance in the presence of time-varying delays}

In this subsection, we compare our system against classic PD+d system proposed in [10] in order to show its unique performance in dealing with the time-varying delays. The time delays in this experiment are approximately $1 \mathrm{~s}$ with $500 \mathrm{~ms}$ variations, and the rate is around 0.5 . We set $\lambda_{1}=0.5, \lambda_{2}=2, \bar{\mu}_{1}=\bar{\mu}_{2}=0.5$, $\bar{\varepsilon}_{1}=\bar{\varepsilon}_{2}=0.5$. In order to guarantee stability, the parameters in the system in [10] are required to satisfy $4 B_{r} B_{l}>\left(T_{1}^{\text {max }^{2}}+\right.$ $\left.T_{2}^{\max ^{2}}\right) K_{l} K_{r}, B_{r} \geq B_{l}$ and $K_{r} \geq K_{l}$. Therefore, we set the differential gain $K_{d}=3$, and the proportional gains $K_{r}=K_{l}=$ 3. Hence, $B_{r}=B_{l}=3.5$. The PD+d system in [10] also uses the scaling gain $\sqrt{1-\bar{\mu}_{1,2}}$ for velocity transmission. It is noticeable that when $\bar{\mu}_{1,2} \geq 1$, this approach is too conservative and velocities cannot be transmitted. Figs. 9 and 10 demonstrate position tracking, position errors and torque tracking of the two systems. The key element in a PD+d system is the velocity damper that can guarantee the system's stability but, instead, degrade the system's transparency.

As shown in Fig.9, with large time delays, the velocity damper $B_{r}$ and $B_{l}$ in the system in [10] have to be set large enough to guarantee stability, and $\sqrt{1-\bar{\mu}_{1,2}}$ also affect the velocity transmission to the extent that the position tracking is affected and large position errors occur. Also, the operator feels the system over-damped and achieves large feedback forces even under free motion.

By contrast, passivity controllers in our system vary based on the observed power signals at each port. Based on Fig.10, the observed power signals are not definitely negative, so that the passivity controllers keep varying between activation and deactivation modes. Therefore, the proposed system is not as conservative as the classical PD+d system in [10] and can achieve more accurate position tracking performance under large timevarying delays. In addition, since the proposed system is not overdamped, the feedback force felt by the operator is not as large as that in the system in [10]. 


\subsection{Performance in the presence of fast-varying delays}

The novelty of the proposed system is also reflected by its ability to deal with fast-varying delays. In this section, we compare the new system with the performance of a system we developed previously [36]. The time delays for the experiment of are set to be very large (around 2 s with 1 s variations) and with a large rate (around 1.5). We set $\lambda_{1}=1, \lambda_{2}=4, \bar{\mu}_{1}=\bar{\mu}_{2}=1.5, \bar{\varepsilon}_{1}=$ $\bar{\varepsilon}_{2}=1$. The slave robots in these two systems are controlled to have a free motion first and then to come in contact with a solid wall in the reverse direction. Figs. 11 and 12 show the position and torque tracking as well as the related tracking errors of the two systems. The previous system [36] primarily has two deficiencies. First, the parameters of this system are seriously restricted by the assumption that $\dot{T}_{1,2}(t) \leq 1$. If $\dot{T}_{1,2}(t)>1$, and the stability of the whole system cannot be guaranteed. The time delay for system in [36] is set to $2 \mathrm{~s}$ with $1 \mathrm{~s}$ variation but at a rate of 0.9 . The related parameters are set as recommended in [36]. The second drawback of system in [36] is how it guarantees system stability by largely reducing the position and torque signals. As shown in Fig.11, the slave robot cannot closely and rapidly track the master robot during free motion, and large torque tracking errors exist during hard contact with the environment.

In contrast, the passivity controllers in the new system are actually velocity dampers with the value varying according to the observed powers. Therefore, according to Fig.12, even with the higher rate of time delays, the position tracking in free motion and torque tracking in hard contact are still better that those of the system in [36]. The experimental results illustrate that our new system is more suited to practical application than the system proposed in [36] for the worst-case scenario
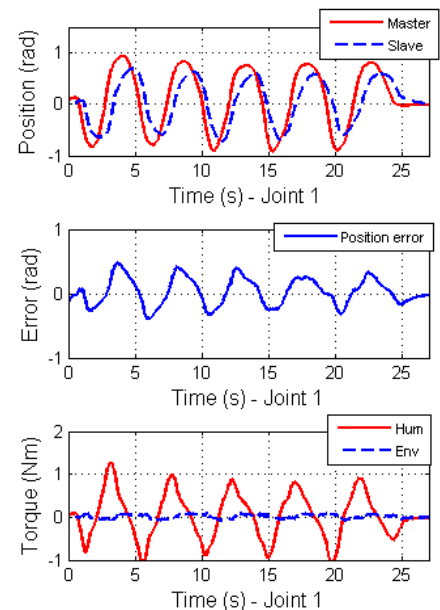
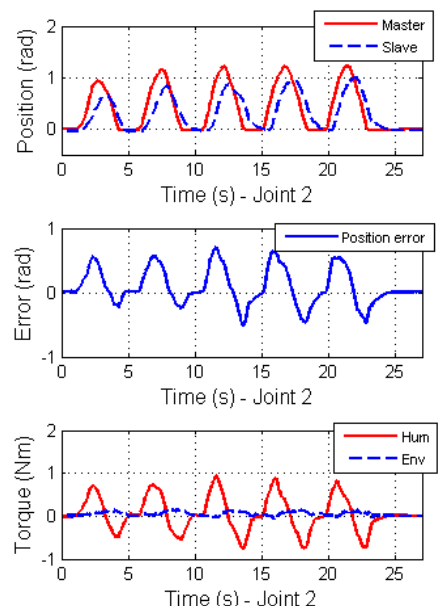
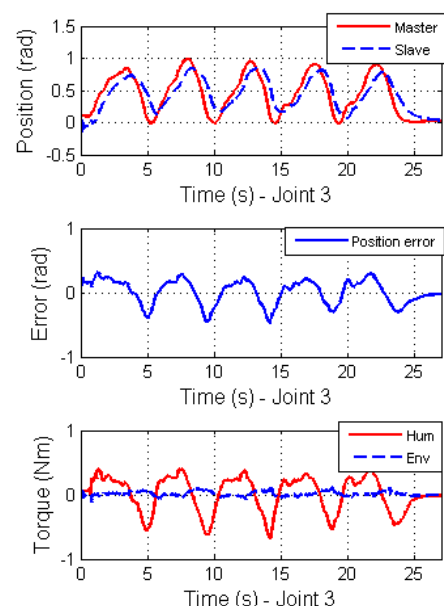

Fig.9. Free motion under time varying delays (PD+d system in [10])
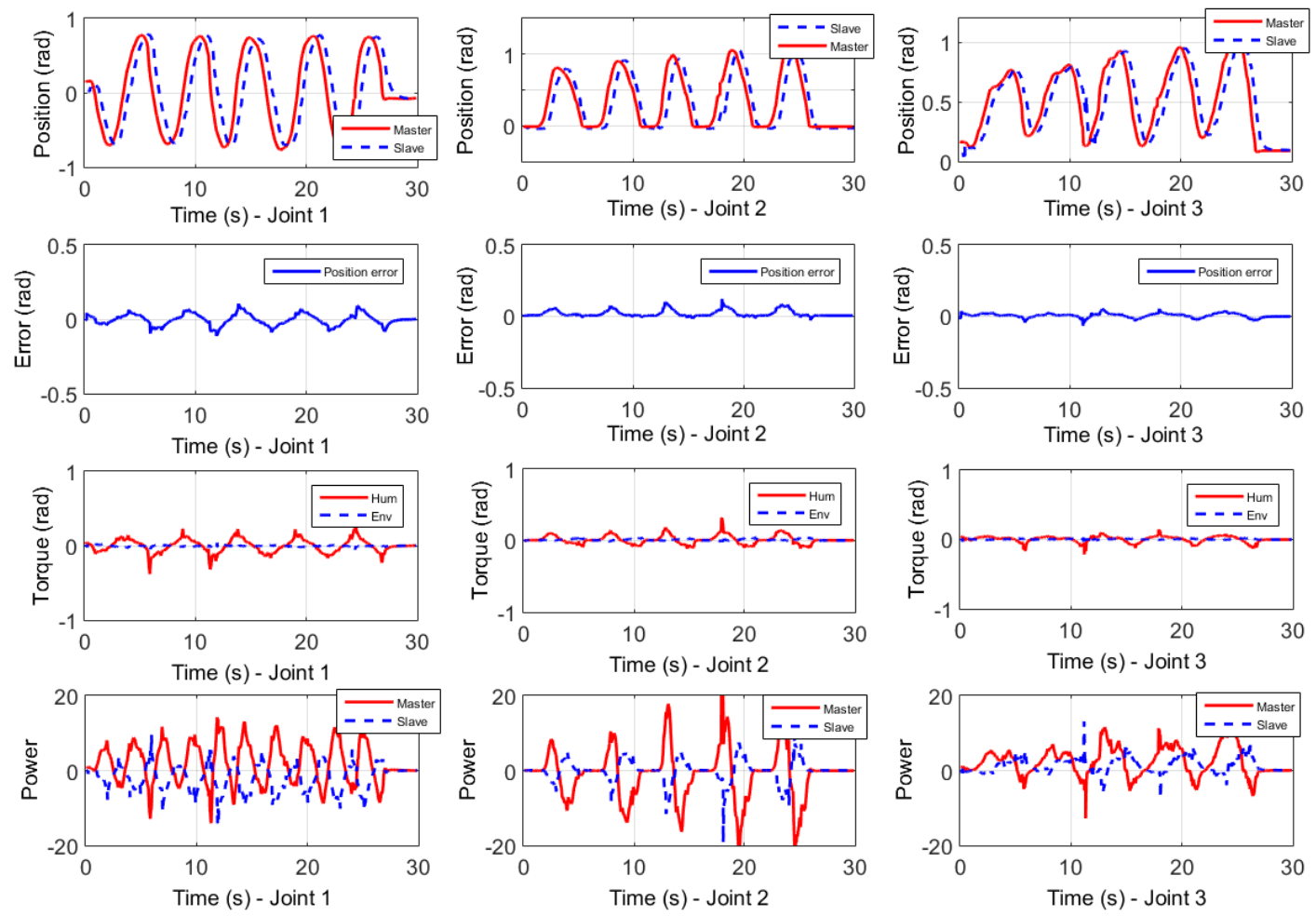

Fig.10. Free motion under time varying delays (our system) 

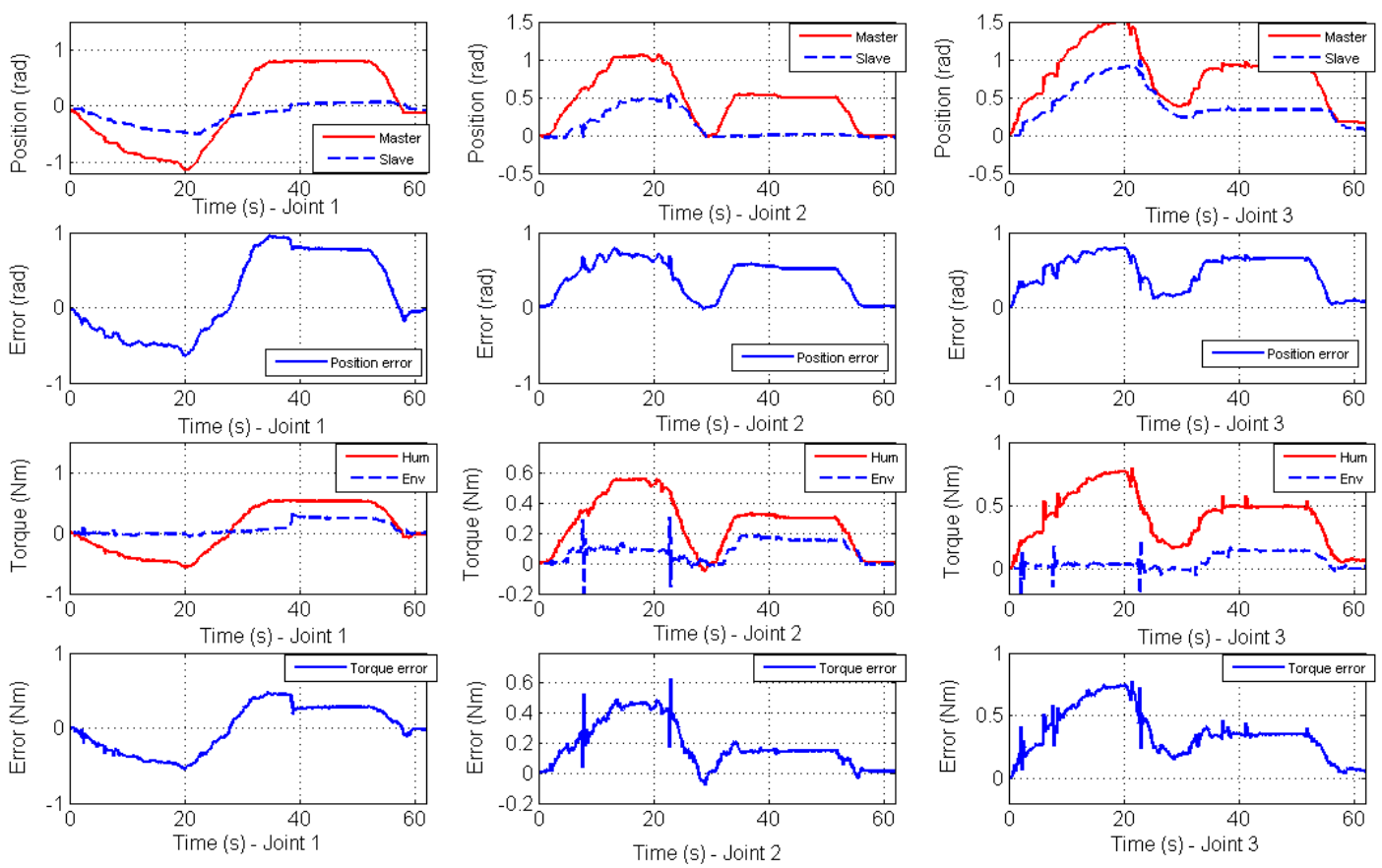

Fig.11. Free motion and hard contact under sharply-varying delays (system in [36])
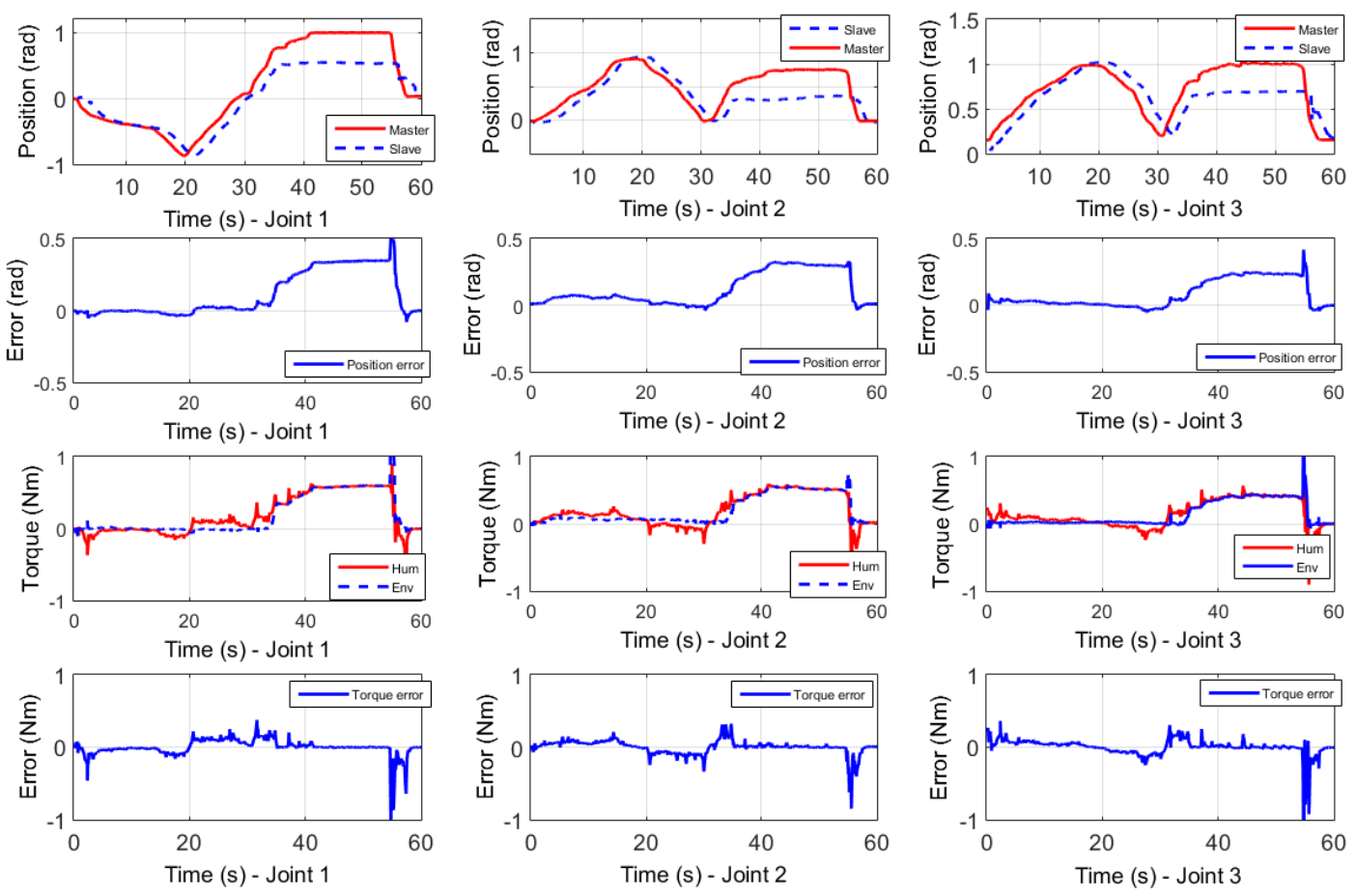

Fig.12. Free motion and hard contact under sharply-varying delays (our system)

\section{Conclusion}

A new 4-CH wave-based TDPA teleoperation system with new passivity observers and controllers was proposed in this paper RBF NN was also deployed to eliminate the nonlinear uncertainties in the dynamic models. Compared with conventional observer, the new observer can more efficiently and accurately monitor the power flow to guarantee that the system is not affected by the passivity controllers under constant time delays. Also, unlike the conventional passivity controllers, the new passivity controllers guarantee the system stability by damping velocity but not reducing position and torque signals. Therefore in the worst case scenario where the rate of time delay is larger than one, the proposed system can still have fine position and torque tracking performances. The system stability with external human and environment inputs was also analyzed using Lyapunov functions. The proposed algorithm was validated using a 3-DOF teleoperation system under different time delay situations, and was shown to be superior over previous algorithm we proposed reported earlier.

Reference: 
[1] Hirzinger, G.; Brunner, B.; Dietrich, J.; Heindl, J., "Sensor-based space robotics-ROTEX and its telerobotic features," in Robotics and Automation, IEEE Transactions on, vol.9, no.5, pp.649-663, Oct 1993

[2] Sheridan, T.B., "Space teleoperation through time delay: review and prognosis," in Robotics and Automation, IEEE Transactions on , vol.9, no.5, pp.592-606, Oct 1993

[3] Hirose, S.; Kato, K., "Development of quadruped walking robot with the mission of mine detection and removal-proposal of shape-feedback masterslave arm," in Robotics and Automation, 1998. Proceedings. 1998 IEEE International Conference on , vol.2, pp.1713-1718 vol.2, 16-20 May 1998

[4] Cragg, L.; Huosheng $\mathrm{Hu}$, "Application of mobile agents to robust teleoperation of internet robots in nuclear decommissioning," in Industrial Technology, 2003 IEEE International Conference on , vol.2, pp.1214-1219

[5] Barnes, B.; Menon, A.S.; Mills, R.; Bruyns, C.D.; Twombly, A.; Smith, J.; Montgomery, K.; Boyle, R., "Virtual reality extensions into surgical training and teleoperation," in Information Technology Applications in Biomedicine, 2003. 4th International IEEE EMBS Special Topic Conference on , pp.142145, 24-26 April 2003

[6] Rybarczyk, Y.; Colle, E.; Hoppenot, P., "Contribution of neuroscience to the teleoperation of rehabilitation robot," in Systems, Man and Cybernetics, 2002 IEEE International Conference on, vol.4, pp.6, Oct. 2002

[7] Quaid, A.E.; Abovitz, R.A., "Haptic information displays for computerassisted surgery," in Robotics and Automation, 2002. Proceedings. ICRA '02. IEEE International Conference on , vol.2, no., pp.2092-2097 vol.2, 2002

[8] Dongjun Lee; Spong, M.W., "Passive Bilateral Teleoperation With Constant Time Delay," in Robotics, IEEE Transactions on , vol.22, no.2, pp.269-281, April 2006

[9] Nuño, E.; Basañez, L.; Ortega, R.; \& Spong, M. W., "Position tracking for non-linear teleoperators with variable time delay," The International Journal of Robotics Research, vol.28, no.7, pp.895-910, 2009

[10] Nuño, E.; Basañez, L.; Ortega, R., "Passivity-based control for bilateral teleoperation: A tutorial." Automatica, vol.47, no.3, pp. 485-495, 2011

[11] Chopra, N.; Mark W. S.; and Rogelio L., "Synchronization of bilateral teleoperators with time delay." Automatica, vol.44, no.8, pp.2142-2148, 2008

[12] Yana, Yang; Changchun Hua; and Xinping Guan; "Coordination control for bilateral teleoperation with kinematics and dynamics uncertainties," Robotics and Computer-Integrated Manufacturing, vol.30, no. 2, pp. 180-188, 2014

[13] Lewis, F.L.; Yesildirek, A.; Kai Liu, "Multilayer neural-net robot controller with guaranteed tracking performance," in Neural Networks, IEEE Transactions on , vol.7, no.2, pp.388-399, March 1996

[14] Ciliz, M. K., "Adaptive control of robot manipulators with neural network based compensation of frictional uncertainties." Robotica vol.23, no.2, pp. 159-167, 2005

[15] Veelenturf L.P.J., "Analysis and applications of artificial neural networks," Prentice-Hall, Inc., 1995.

[16] Yang, Y., Ge, C., Wang, H., Li, X., \& Hua, C, "Adaptive neural network based prescribed performance control for teleoperation system under input saturation." Journal of the Franklin Institute vol.352, no.5, pp. 1850-1866 2015

[17] Yang, Y., Hua, C., and Guan, X.. "Finite Time Control Design for Bilateral Teleoperation System With Position Synchronization Error Constrained." Cybernetics, IEEE Transactions on, online first, 2015.

[18] Forouzantabar, A., Talebi, H. A., \& Sedigh, A. K., "Adaptive Neural Network Control of Bilateral Teleoperation with Unsymmetrical Stochastic Delays and Unmodelled Dynamics", International Journal of Robust and Nonlinear Control, vol.24, no.11, pp.1628-1652, 2012

[19] Li, Z., \& Su, C. Y., "Neural-adaptive Control of Single-master multiple slaves Teleoperation for Coordinated Multiple Mobile Manipulators with Time-varying Communication Delays and Input Uncertainty", IEEE

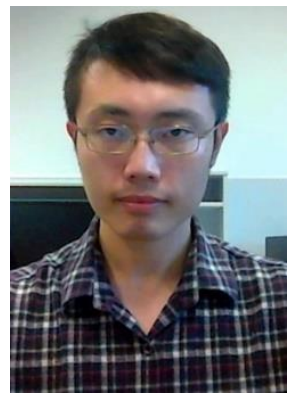

Da Sun received the B.Sc. degree in telecommunication engineering from the University of Wollongong, Wollongong, N.S.W, Australia, in 2012, where he is currently working toward the Ph.D. degree in electrical engineering. His research interests include robotics and teleoperation systems
Transactions on Neural Networks and Learning Systems, vol. 24 , no. 9, pp. 1400-1413, Sept. 2013

[20] Li, Z.; Xia, Y.; Wang, D.; Zhai, D.-H.; Su, C.-Y.; Zhao, X., "Neural NetworkBased Control of Networked Trilateral Teleoperation With Geometrically Unknown Constraints," in Cybernetics, IEEE Transactions on, In publication process

[21] Li, Z., Xia, Y., \& Su, C. Y, “Intelligent Networked Teleoperation Control," Springer, 2015

[22] Yokokohji, Y., Imaida, T., \& Yoshikawa, T., "Bilateral teleoperation under time-varying communication delay," IEEE/RSJ International Conference, vol.3, pp.1854-1859, 1999

[23] Munir, S.; Book, W.J., "Internet-based teleoperation using wave variables with prediction," in Mechatronics, IEEE/ASME Transactions on , vol.7, no.2, pp.124-133, Jun 2002

[24] Yongqiang Ye; Liu, P.X., "Improving Haptic Feedback Fidelity in WaveVariable-Based Teleoperation Orientated to Telemedical Applications," inInstrumentation and Measurement, IEEE Transactions on, vol.58, no.8, pp.2847-2855, Aug. 2009

[25] Li, Hongbing, \& Kenji Kawashima. "Achieving Stable Tracking in WaveVariable-Based Teleoperation." IEEE Transactions on Mechatronics, Vol. 19 No. 5, pp. 1574-1582, Oct. 2014.

[26] Chan, Linping, Fazel Naghdy, David Stirling. "Extended active observer for force estimation and disturbance rejection of robotic manipulators." Robotics and Autonomous Systems vol.61, no.12 pp. 1277-1287, 2013.

[27] Yongqiang Ye; Liu, P.X., "Improving Trajectory Tracking in Wave-VariableBased Teleoperation," in Mechatronics, IEEE/ASME Transactions on, vol.15, no.2, pp.321-326, April 2010

[28] Aziminejad, A.; Tavakoli, M.; Patel, R.V.; Moallem, M., "Transparent TimeDelayed Bilateral Teleoperation Using Wave Variables," in Control Systems Technology, IEEE Transactions on , vol.16, no.3, pp.548-555, May 2008

[29] Bate, L., Cook, C. D., \& Li, Z. "Reducing Wave-Based Teleoperator Reflections for Unknown Environments," Industrial Electronics, IEEE Transactions on , vol. 58, no. 2, pp.392-397, 2011.

[30] Sun, D., Naghdy, F., \& Du, H., "Transparent four-channel bilateral control architecture using modified wave variable controllers under time delays", Robotica, pp.1-17, 2014

[31] Ryu, J. H., Kwon, D. S., \& Hannaford, B., "Stable teleoperation with time domain passivity control," IEEE Trans. Robot. Autom., vol. 20, no. 2, pp. 365-373, Apr. 2004.

[32] Artigas, J., Ryu, J. H., \& Preusche, C., Time domain passivity control for position-position teleoperation architectures. Presence: Teleoperators and Virtual Environments, vol.19, no.5, pp.482-497, 2010

[33] Ye, Y., Pan, Y. J., Gupta, Y., \& Ware, J. "A power-based time domain passivity control for haptic interfaces." Control Systems Technology, IEEE Transactions on vol.19, no.4, pp.874-883, 2011

[34] Ye, Y., Pan, Y. J., \& Hilliard, T., "Bilateral teleoperation with time-varying delay: A communication channel passification approach," IEEE/ASME Trans. Mechatronics, vol. 18, no. 4, pp. 1431-1434, Aug. 2013.

[35] Chawda, V.; OMalley, M.K., "Position Synchronization in Bilateral Teleoperation Under Time-Varying Communication Delays," Mechatronics, IEEE/ASME Transactions on, vol.20, no.1, pp.245,253, Feb. 2015.

[36] Sun, D., Naghdy, F., \& Du, H., "Wave-variable-based Passivity Control of Four-channel Nonlinear Bilateral Teleoperation System under Time Delays." Mechatronics, IEEE/ASME Transactions on, online first, 2015.

[37] Chang-Chun Hua; Liu, X.P., "Delay-Dependent Stability Criteria of Teleoperation Systems With Asymmetric Time-Varying Delays," in Robotics, IEEE Transactions on , vol.26, no.5, pp.925-932, Oct. 2010

[38] Chang-Chun Hua; Yana Yang; Xinping Guan, "Neural network-based adaptive position tracking control for bilateral teleoperation under constant time delay." Neurocomputing vol.113, pp.204-212, 2013.

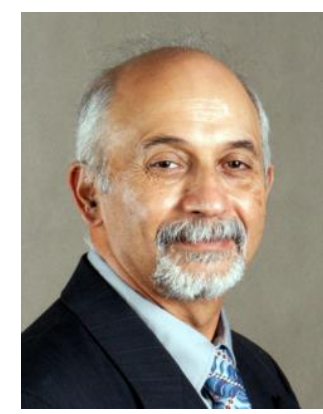

Fazel Naghdy has a demonstrated track record and leadership in research, teaching, and management. His research has had its focus on machine intelligence and control particularly in embedded mechatronics and robotics systems. He is also contributing reviewer to IEEE Transactions on Mechatronics Engineering, and International Journal of Intelligent Automation and Soft Computing, and many others. He is the Director of Centre for Intelligent Mechatronics Research. His current research interests 
include haptic rendered virtual manipulation of clinical and mechanical systems, intelligent control and learning in non-linear and non-structured systems. He is currently a Professor of Robotics and Intelligent Systems at University of Wollongong.

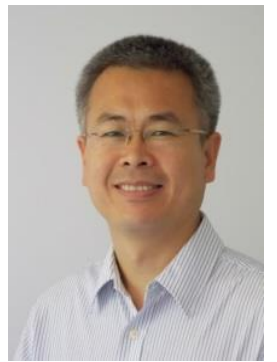

Haiping Du received his Ph.D. degree in mechanical design and theory from Shanghai Jiao Tong University, Shanghai, PR China, in 2002. He was Research Fellow in University of Technology, Sydney from 2005-2009, and Post-Doctoral Research Associate in Imperial College London (2004-2005) and the University of Hong Kong (2002-2003). He is currently Associate Professor of School of Electrical, Computer \& Telecommunications Engineering, University of Wollongong. His research interests include vibration control, vehicle dynamics and control systems, robust control theory and engineering applications, electric vehicles, robotics and automation, smart materials and structures. He is a recipient of the Australian Endeavour Research Fellowship (2012). 